\title{
UK Breast Cancer Research Symposium 2016: Submitted Abstracts
}

(C) The Author(s) 2016. This article is published with open access at Springerlink.com

\section{Breast cancer risk alleles in separate gene regulatory elements across $6 q 25$ regulate the ESR1, RMND1 and $\mathrm{CCDC170}$ genes and alter breast tumour traits}

\author{
Alison Dunning, Edwards S., Michailidou K., Kuchenbaecker K, \\ Thompson D., Lopez-Knowles E., Dowsett M., \\ Pharoah P., Garcia-Closas M., Chene
}

Centre for Cancer Genetic Epidemiology, Cambridge University

Genetic variants at $6 \mathrm{q} 25$ are associated with breast cancer in the general population and in BRCA1 mutation carriers. To identify the causal variants underlying these associations, we analysed 3872 SNPs across $6 \mathrm{q} 25$ in 118,816 subjects from three international consortia. We observed five separate regions of association-surrounding ESR1- the most obvious target gene in the region. We used epidemiological methods to define the best causal candidate variants and examined their phenotypic associations:

At four of the five regions, the causal candidate risk alleles display a stronger association with oestrogen receptor-negative (ER-) than oestrogen receptor-positive (ER+) tumours. Three of these four are associated with "triple-negative" and the fourth with HER2+ tumour subtypes. Two are additionally associated with mammographic density. The candidate causal variants in the fifth region are more strongly associated with high-grade ER+ breast tumours.

These causal candidates lie in five cis-regulatory elements. Chromosome conformation capture confirmed that these elements directly contact the promoters of the ESR1, RMND1, ARMT1 and CCDC170 genes. In the ER-associated regions, the best causal candidates overlap four separate enhancers and reporter assays indicated their risk alleles putatively reduced expression of ESR1, RMND1 and CCDC170. These findings were validated using immunohistochemical and other studies. Luciferase assay constructs carrying the causal candidate risk alleles decreased ESR1, RMND1 and CCDC170 promoter activity. By contrast, the risk alleles most strongly associated with ER+ tumour risk disrupt a silencer element and increase ESR1 and RMND1 expression.

Risk alleles disrupting the enhancer elements reduce oestrogen receptor expression and increase the risk of ER- tumour subtypes, while disrupting the silencer element increases oestrogen receptor expression and risk of high-grade ER+ tumours, suggesting there may be a "Goldilocks level" of ESR1 expression for breast cancer protection.

\section{Disrupting coordinated cytoskeletal networks inactivates cancer-associated fibroblasts and deters breast cancer progression}

Fernando Calvo, Romana Ranftl, Steven Hooper, and Erik Sahai

The Institute of Cancer Research, London

Cancer-associated fibroblasts (CAFs) are non-malignant cells prominently found in human breast cancers. CAFs can favour tumour progression, dissemination and therapeutic resistance through remodelling of the extracellular matrix and signalling to cancer, endothelial and immune cells. Targeting CAFs represents an alternative for therapeutic intervention. Our goal is to identify crucial mechanisms governing the tumour-promoting phenotype of CAFs that, once targeted, inactivate CAFs and deter tumour progression.

We isolated fibroblasts from different stages of breast cancer progression and normal counterparts, and analysed their phenotype, function and gene expression. Our analyses revealed that enhanced mechano-transduction and activation of the transcriptional regulator YAP are signature features of CAFs. YAP regulates the expression of cytoskeletal genes that are required for the maintenance of the CAF phenotype and for their ability to remodel the extracellular matrix and promote cancer cell invasion and angiogenesis. Matrix stiffening further enhances YAP activation, thus establishing a feed-forward self-reinforcing loop that helps to maintain the CAF phenotype. In addition, we identified Cdc42EP3, a previously uncharacterised $\mathrm{Cdc} 42$ effector, as a key regulator of CAFs. Cdc42EP3 links actin and septin fibres and is upregulated early in fibroblast activation. Cdc42EP3 induces a profound cytoskeletal rearrangement in CAFs that enables mechano-transduction and the emergence of pro-tumorigenic functions, which are also dependent on the presence of a septin fibrillary network.

Our studies show how mechanical inputs in solid tumours modulate the aggressive behaviour of stromal fibroblasts. We demonstrate a critical role for coordination between the actin and the septin networks in CAFs that is required for mechano-transduction, forcemediating matrix remodelling and promoting cancer cell invasion, angiogenesis and tumour growth. Finally, we describe the mechanism of action of Cdc42EP3 and define a novel molecular and biological role for septins in CAFs. 


\section{Patient-derived xenograft models of breast cancer with human immune components}

\section{Rebecca Marlow ${ }^{1}$, Kristina Ilieva ${ }^{1,2}$, Fernanda Kyle ${ }^{3}$, Erika Francesch $^{1}$, Panagiotis Karagiannis ${ }^{2}$, Patrycja Gazinska, Frank Nestle, Adrian Hayday ${ }^{3}$, Sophia Karagiannis ${ }^{1,2}$, Andrew Tutt ${ }^{1}$}

${ }^{1}$ Breast Cancer Now Research Unit, Division of Cancer Studies, Faculty of Life Sciences and Medicine, King's College London SE1 9RT, UK; ${ }^{2}$ Skin Science, Division of Genetics and Molecular Medicine, Faculty of Life Sciences and Medicine, King's College London SE1 9RT, UK; ${ }^{3}$ Peter Gorer Department of Immunobiology, Division of Immunology, Infection \& Inflammatory Disease, Faculty of Life Sciences and Medicine, King's College London SE1 9RT, UK

Background It is now recognised that many cancers, including breast, elicit immune cell engagement resulting in response directed against overexpressed or aberrantly presented antigens. A greater understanding of these interactions has significantly improved prognosis in other tumor types. Here we aim to build disease-relevant in vivo models of breast cancer that recapitulate the immune tumour microenvironment. Patientderived xenografts (PDX) are in vivo preclinical models derived from transplantation of patient tumour into immunocompromised hosts. These models recapitulate major clinicopathologic features of patient tumours. However, as they are maintained in immunocompromised hosts, we cannot study interactions between tumour and immune cells. In order to address this, we established humanised breast cancer PDX models. Method We developed humanised TNBC PDX models in NSG mice, based on those used in melanoma. The PDX tumour is established by orthotopic transplantation. Host is then engrafted with human immune effector cells (peripheral blood lymphocytes (PBL) from healthy volunteers or matched patient). Following tumour development, tissues are processed for analysis.

Results We observe 40\% engraftment of human immune effector cells in host spleens following tumor development by flow analysis. We also observe human CD45 + leukocyte populations in the tumour, blood and other organs. Tumour-infiltrated lymphocytes (TIL) expressed the immune checkpoint phenotype of patient cancers. We are now investigating the subtypes of TIL present in our model, whether novel antibody-based therapies can be used to block tumour formation, and how immune checkpoints are modulated.

Conclusion Our overarching aim is to determine whether these models recapitulate immune responses directed against tumor antigens in order to study mechanisms of action and efficacy of novel therapeutics to treat breast cancer. Preclinical humanised PDX models may be feasible approaches to accelerate therapeutic discovery relevant to impacting tumour and immune stromal interaction for patient benefit.

\section{Long-term neoadjuvant endocrine treatment as a clinical model of breast cancer dormancy}

\author{
Cigdem Selli, Arran K. Turnbull, Renshaw L., \\ Thomas J.S., J. Michael Dixon, Andrew H. Sims
}

Applied Bioinformatics of Cancer, Edinburgh Cancer Research Centre, Institute of Genetics and Molecular Medicine, The University of Edinburgh

Aim A minority of oestrogen receptor (ER)-positive breast tumours treated with neoadjuvant aromatase inhibitors maintain a stable size by becoming dormant and continue to receive extended treatment and therefore represent the best currently available clinical model to investigate dormancy. We aimed to determine genome-wide expression profiles of dormant cancer cells from breast tumour biopsies that have received extended neoadjuvant letrozole.

Patients and methods All patients were classified as "responders" based upon ultrasound measurements after 3 months of letrozole treatment and did not have a recurrence by the latest biopsy. Expression analysis was performed using Illumina BeadChips. R and BioConductor packages were used for analysis. Differentially expressed genes were determined using paired Rank Products (FDR, $5 \%$ ). Pathway analysis was performed using Panther database.

Findings A total of 13 patient-matched 'dormant' ( $>6$ months treatment) and baseline samples were compared with each other and earlier on-treatment biopsy samples. A total of 358 genes were significantly differentially regulated ( 90 down, 268 up) between paired baseline and long-term treatment samples from patients with 'dormant' tumours. A subset of genes (79 down, 255 up) were found to be uniquely altered during long-term letrozole treatment, and functional analysis revealed that they were enriched for a number of cancerrelated pathways, including angiogenesis, cadherin signalling, cell cycle and blood vessel development. Changes in gene expression in patients that have recurrences under long-term letrozole treatment will be determined and further compared with dormancy signature. Conclusion This is the first patient-matched gene expression study to look at aromatase inhibitor-induced dormancy in breast cancer. Our results may help to characterise extended growth suppression in letrozole-treated dormant-state breast cancer cells.

\section{Synthetic lethality and viability: how does BRCA1 interact with 53BP1?}

\section{Jo Morris}

Institute of Cancer and Genomic Sciences, University of Birmingham

Whether or not the intrinsic activity of the breast and ovarian cancer predisposition protein BRCA1 as an E3 ubiquitin ligase plays any part in suppressing genomic instability has been controversial. Through mutation of a novel element within the dimeric RING domains of the BRCA1:BARD1 complex, we demonstrate the ligase activity is required for a subset of BRCA1 functions in the damage response. Significantly, we show that the ubiquitin ligase activity is a key determinant of how BRCA1 counters the inhibitory influence of 53BP1 on DNA resection to promote homologous recombinational DNA repair. Evidence indicating how this is achieved will be presented. The highest density of patient missense changes are found within the region of $B R C A 1$ that directs ligase activity, and our findings indicate mutational status will be significant in therapy choice and resistance mechanisms associated with synthetic viability. More widely, these findings functionally illustrate the multifaceted role of BRCA1 in the DNA damage response, suggesting new approaches to overcome resistance mediated by loss of $53 \mathrm{BP} 1$ or $53 \mathrm{BP} 1$ effector pathways.

\section{Functional characterisation of low-frequency mutations in breast cancer}

Andri Leonidou, Sarah L. Maguire, Patty T. Wai, Paul Huang, Barrie Peck and Rachael C. Natrajan

The Institute of Cancer Research, London

Next-generation sequencing efforts have unveiled a vast complexity in the mutational repertoire of breast cancer. Aside highly recurrent 
mutations in known driver genes such as TP53 and PIK3CA, the vast majority of mutations occur at low frequencies in unselected breast cancers and remain uncharacterised.

Our aims were to functionally investigate the significance of potentially oncogenic mutations in breast cancer in order to identify novel drivers and therapeutic targets. To this end, we compiled a database of mutations identified from publicly available and in-house breast cancer sequencing data. High-confidence variants were run through bioinformatic prediction algorithms FATHMM and CHASM to assess their oncogenic impact, and CANSAR was used to annotate the genes which are druggable. Following several exclusion criteria and manual curation, the list was limited to 22 missense mutations across 8 tyrosine kinases.

Mutant and wild-type cDNA constructs were transduced into a panel of cell lines in order to characterise the phenotypic impact of mutations using in vitro $2 \mathrm{D}$ and $3 \mathrm{D}$ assays.

We found FGFR2 hotspot mutations to be constitutively active, showing a high global phosphorylation state consistent with previous reports. Cells carrying this mutation were sensitive to siRNA-mediated silencing of FGFR2 and chemical inhibition with AZD4547 and PD173074. INSRR demonstrated a context-dependent phenotype, and its expression has been correlated with outcome. Kinase domain mutations conferred a proliferative advantage when cells were grown in $2 \mathrm{D}$ culture. This was more pronounced in 3D spheroids, suggesting that features such as cell-cell contact, oxygen and $\mathrm{pH}$ gradients across the spheroids better recapitulate physiological conditions and present a more appropriate system for the assessment of moderate-impact drivers.

Overall, we found that some lower frequency kinase mutations are oncogenic. However, the impact of some of these can only be fully understood in conditions that more closely resemble in vivo conditions, such as $3 \mathrm{D}$ culture.

\section{Modelling the tumour microenvironment and employing functional genomics identifies CREBBP as a novel tumour surpressor in triple negative breast cancers}

\section{Barrie Peck, Sarah Maguire, Eamonn Morrison, Patty Wai, Rachael Natrajan}

\section{The Institute of Cancer Research, London}

Recent next-generation sequencing studies have comprehensively mapped the genetic landscape of breast cancer and revealed that only a small number of genes are recurrently mutated in more than $10 \%$ of unselected tumours (i.e. TP53, PIK3CA and GATA3), and that the vast majority of recurrent mutations occur at low frequencies. Although some have been shown to be drivers, i.e. confer a selective advantage, there are a myriad of significantly altered lower frequency mutations whose functional impact is unknown. We utilized a functional genomics approach silencing the 200 most frequently mutated genes in breast cancer in 3-dimensional (3D) spheroid cultures, using the MCF10a progression series cell line panel to identify novel lossof-function mutations that affect breast cancer progression from nonmalignant to highly invasive disease.

Using cancer cell spheroids to more accurately recapitulate in vivolike conditions, we identified 11 genes whose silencing with siRNA impacted growth in two or more cell lines in 3D. Furthermore, a second targeted validation screen showed that silencing of a cohort of these genes had limited effect under traditional 2D culture conditions. The silencing of the histone acetyltransferase CREBBP promoted growth in AT1, DCIS.com and CA1H cells in 3D while having no effect in 2D cultures. Investigation of The Cancer Genome Atlas (TCGA) showed that CREBBP was more frequently mutated in triple-negative breast cancers (TNBCs). Moreover, at least a third of TNBCs also displayed haploinsufficient loss of CREBBP and this loss resulted in the upregulation of the pro-proliferative transcription factor FOXM1. Multivariate analysis of CREBBP expression showed that CREBBP loss was significantly associated with decreased relapse-free survival.

In summary, using a functional genomics approach in 3D models we found that CREBBP silencing promotes growth in tumour spheroids. Furthermore, CREBBP activity is altered in a significant proportion of TNBCs and associated with decreased relapse survival.

\section{Synthetic lethal approaches to target ARID1A deficient breast cancer}

Saira Khalique, C.T. Williamson, H Pemberton, P. T. Wai, M. Menon, R. Brough, A. Leonidou, B. Peck, R. C. Natrajan and C. J. Lord

The Institute of Cancer Research, London

Dysregulation of the SWI/SNF complex is one of the most commonly occurring defects in solid cancers; in many cases, SWI/SNF defects are caused by loss-of-function mutations in the tumour suppressor ARID1A (AT-rich interactive domain-containing protein 1A). Mutations in ARID1A occur in around $4 \%$ of unselected breast cancers, the vast majority being loss-of-function frameshift or nonsense mutations, resulting in loss of protein function. In addition, loss of ARID1A expression is a common occurrence in breast tumours, especially in high-grade tumours, and is correlated with a poor prognosis.

Despite the understanding that ARID1A defects are associated with tumourigenesis, targeted therapy approaches that exploit this deficiency have not as yet been developed. To this end, we have devised a large-scale functional genomics approach to identify actionable ARID1A synthetic lethal effects. Using a high-throughput drug screen with a plate library of 80 compounds that are currently licenced or in early phase trials, in isogenic ARID1A null and wildtype MCF10A cells, we have identified a series of novel ARID1A synthetic lethal effects. Subsequent drug combination studies have identified candidate therapeutic approaches to targeting ARID1A mutant tumours that could be assessed in proof-of-concept clinical trials. Assessment of this combinatorial approach in in vivo models of ARID1A mutant cancers is now underway. In conclusion, we have identified clinically actionable combinatorial approaches that may provide additional therapeutic benefit for ARID1A deficient patients.

\section{PIM1 kinase regulates cell death, tumour growth and chemotherapy resistance revealing a novel target in triple-negative breast cancer}

Fara Brasó-Maristany ${ }^{1,2}$, Simone Filosto ${ }^{1,2}$, Steven Catchpole ${ }^{1,2}$, Rebecca Marlow ${ }^{1,2}$, Jelmar Quist ${ }^{1,2}$, Erika Francesch Domenech $^{1,2}$, Darren A. Plumb ${ }^{1,2}$, Leila Zakka ${ }^{1,2}$, Patrycja Gazinska $^{1,2}$, Gianmaria Liccardi ${ }^{3}$, Pascal Meier ${ }^{3}$, Albert GrisOliver $^{4}$, Maggie Chon U. Cheang ${ }^{3}$, Anna Perdrix Rosell ${ }^{1,2}$, Manar Shafat $^{1,2}$, Elodie Noël ${ }^{1,2}$, Nirmesh Patel ${ }^{1,2}$, Kristen McEachern ${ }^{5}$, Maurizio Scaltriti ${ }^{6,7}$, Pau Castel ${ }^{6,7}$, Farzana Noor ${ }^{1,2}$, Richard Buus $^{3}$, Sumi Mathew ${ }^{1,2}$, Johnathan Watkins ${ }^{1,2}$, Violeta Serra ${ }^{4}$, Pierfrancesco Marra ${ }^{1,2}$, Anita Grigoriadis ${ }^{1,2}$, Andrew N. Tutt ${ }^{1,2,3}$

${ }^{1}$ Breast Cancer Now Research Unit, King's College London, UK; ${ }^{2}$ Department of Research Oncology, King's Health Partners AHSC, 
Faculty of Life Sciences and Medicine, King's College London, UK; ${ }^{3}$ The Breast Cancer Now Toby Research Centre, The Institute of Cancer Research, London, UK; ${ }^{4}$ Experimental Therapeutics Group, Vall d'Hebron Institute of Oncology, Barcelona, Spain; ${ }^{5}$ Oncology iMed, AstraZeneca, Waltham, Massachusetts, USA; ${ }^{6}$ Department of Medicine, Memorial Sloan Kettering Cancer Center, New York, USA; ${ }^{7}$ Department of Pathology, Memorial Sloan Kettering Cancer Center, New York, USA

Background Triple-negative breast cancers (TNBCs) have poor prognosis and lack targeted therapies, mainly due to the absence of suitable molecular targets. The genomic region 6 p21-p25 is recurrently amplified in TNBCs and encompasses the PIM1 oncogene. PIM1 belongs to the PIM kinase family, implicated in the control of cancer cell proliferation, apoptosis and migration, particularly in leukaemia and prostate cancer. The activity of PIM1 in these cancers has been closely linked to the regulation of c-MYC, an untargetable oncogene frequently amplified and overexpressed in primary and in chemotherapy-resistant TNBCs.

Methods Genomic breast cancer datasets were interrogated to establish PIM1 copy number and transcript levels. To elucidate the role of PIM1 in malignant phenotypes of TNBCs, RNA interference and rescue-of-function experiments were carried out in breast cancer and non-malignant mammary epithelial cell line models. The therapeutic potential of targeting PIM1 in vitro and in vivo was assessed using AZD1208, a small-molecule inhibitor of the PIM kinase family.

Results Interrogation of genomic datasets identified increased PIMI copy number-driven expression in TNBC. Functional studies revealed addiction to PIM1 kinase for cell population growth and apoptosis protection in TNBC cells, absent in non-malignant cells. PIM1 knockdown reduced BCL2 expression, and dynamic BH3 profiling analysis revealed that PIM1 prevents mitochondrial-mediated apoptosis in TNBC cell lines. PIM1 expression was associated with several MYC transcriptional signatures and promoted cell population growth through regulation of c-MYC and transcription of MYC targets, including MCL1. We further demonstrated PIM1 regulation of established TNBC oncogenic proteins SHP2 and EPHA2 and cell cycle inhibitor p27. AZD1208 inhibited growth and sensitised TNBC cell lines, xenografts and patient-derived xenografts to standard-of-care chemotherapy.

Conclusion Targeting PIM1 is a very promising therapeutic strategy for TNBC, a particularly challenging and often chemotherapy-resistant subtype that currently lacks targeted therapies.

\section{Identification of novel genetic dependencies of SF3B1 driver mutations in breast cancer}

Patty Wai, Sarah L. Maguire, Andri Leonidou, Helen Pemberton, Barrie Peck, Christopher J. Lord and Rachael Natrajan

\section{The Institute of Cancer Research}

Mutations in SF3B1 that are involved in RNA splicing have been found to occur at relatively high frequencies in several tumour types and have been postulated as bona fide drivers. Our aims were to investigate whether dysfunction in RNA splicing is implicated in SF3B1 mutant breast cancers and whether tumours harbouring these mutations could be therapeutically targeted.

Analysis of published exome, whole-genome and RNA-sequencing data of $>1000$ primary tumours revealed hotspot mutations in SF3B1 are preferentially associated with ER-positive disease (2\%) including a higher frequency (up to $16 \%$ ) in some rare histological subtypes. Massively parallel RNA sequencing identified consistent patterns of aberrant splicing in SF3B1 mutant tumours that are validated in a re-analysis of RNA-sequencing data from The Cancer Genome Atlas (TCGA). Analysis of reverse phase protein array data from primary tumours demonstrated that mutant tumours show perturbation of common oncogenic signalling pathways, such as AKT and mTOR, rather than a dependency of mutant cells to individual alternatively spliced transcripts. Importantly, cells harbouring SF3B1 mutations were selectively sensitive to SF3b spliceosome complex inhibitors, and treatment of mutant cells resulted in reversal of the aberrant RNA splicing signature.

We further investigated whether SF3B1 could be exploited therapeutically by performing a high-throughput drug screen utilising isogenic cell lines harbouring the SF3B1 K700E hotspot mutation. This identified a number of lead compounds that selectively killed mutant cells, including the AKT and mTOR inhibitors, further highlighting the importance of these signalling pathways in mutant tumours.

Our findings indicate that mutations in SF3B1 are potentially therapeutically tractable in breast cancers, and have identified a number of potential genetic dependencies of oncogenic signalling pathways in SF3B1 mutant tumours.

\section{Survival and costs of postmastectomy radiotherapy among N1 breast cancer patients: A propensity score- matched study}

Chihtao Cheng, Skye Hung-Chun Cheng, MD, Zhen-Ying Liu, MPH, Yi-Ping Lin, MS, Lay-Chin Lim, MS

\section{Koo Foundation Sun Yat-sen Cancer Center}

Purpose/Objectives The St. Gallen Breast Cancer Conference 2015 found no consensus regarding the application of postmastectomy radiotherapy (PMRT) in N1 breast cancer patients. This study examined the effect of PMRT on the survival and medical costs of N1 mastectomy breast cancer patients.

Materials/Methods Taiwan Cancer Registry data and National Health Insurance claims data were used to identify female patients who were newly diagnosed with pT1-2N1 breast cancer between 2004 and 2009. Exclusion criteria included diagnosis of bilateral breast cancer or metastatic disease, positive surgical margins, and radiotherapy or chemotherapy before mastectomy. Of the 6422 patients who met the inclusion criteria, 2926 (46\%) had PMRT and 3496 $(54 \%)$ did not. One-to-one propensity score matching was used to control for potential confounding factors including age, comorbidities, pathological $\mathrm{N}$ and $\mathrm{T}$ stages, tumor grade, year of diagnosis, and having received chemotherapy or hormone therapy.

Results In Kaplan-Meier analysis, we found the 5-year overall survival rates and disease-free survival rates in PMRT group were higher than non-PMRT group $(92.1 \%$ vs. $90.1 \%, \mathrm{p}=0.0072$ and $79.3 \%$ vs. $73.8 \%, \mathrm{p}<0.0001)$, respectively. The PMRT group had significantly higher overall survival (hazard ratio $(\mathrm{HR})=0.79$; $\mathrm{p}=0.0177 ; 95 \% \mathrm{CI}=0.643-0.959)$ and disease-free survival $(\mathrm{HR}=0.75 ; \mathrm{p}<0.001 ; 95 \% \mathrm{CI}=0.664-0.857)$ compared with the non-PMRT group. A mean net increase of 0.10 year in overall survival and 0.43 year in disease-free survival was observed. The mean cost totals were US $\$ 16,559$ for the non-PMRT group and US $\$ 20,778$ for the PMRT group. An incremental cost-effectiveness ratio of US\$42,190 for overall survival and US\$9812 for disease-free survival per year gained was found in our analysis.

Conclusions PT1-2N1 breast cancer patients treated with PMRT exhibited superior survival outcomes and an acceptable cost-effectiveness ratio. 


\section{Ganoderma applanatum - potential target for stimulating macrophages in immunosuppressive breast cancer microenvironment}

\author{
Javed S. ${ }^{1}$, Payne G.W ${ }^{2}$, Lee C.H. ${ }^{1}$ \\ ${ }^{1}$ Biochemistry \& Molecular Biology, ${ }^{2}$ Northern Medical Program, \\ University of Northern British Columbia, Canada
}

Natural products have been used as main ingredients of traditional medicines for thousands of years. Breast cancer is the most common cancer affecting women worldwide. Problems such as toxicity, resistance, and nonspecificity of the target limit the use of chemotherapy. This dilemma calls for the need to explore natural ways to cure cancer by activating the immune system.

Macrophages, major immune cells, are one of the most abundant and vital key players in the breast cancer microenvironment. They play a critical role in each step from tumor initiation to its progression and development. In the initial antitumour response, these macrophages release pro-inflammatory cytokines such as interleukin 6 (IL$6)$, tumour necrosis factor alpha (TNF- $\alpha$ ), etc., which are helpful in destroying breast cancer cells. However, in chronic inflammatory stages, these macrophages get switched to tumour-associated macrophages (TAMs) which are more inclined towards anti-inflammatory and immunosuppressive response, consequently inhibiting the tendency of the body to fight against the tumours. These suppressed macrophages could be a potential target for anticancer therapy as, with the help of stimulation, these can regain their power to kill tumour cells.

The current study aims to investigate the effects of Ganoderma applanatum, a wild mushroom of British Columbia, Canada. This mushroom experienced sequential extraction with the help of four solvents: $80 \%$ ethanol, $50 \%$ methanol, water, and $5 \% \mathrm{NaOH}$. These extracts were then tested on RAW 264.7 macrophage cells for their ability to stimulate TNF- $\alpha$ production. The results showed that water extract of Ganoderma applanatum demonstrated the most effective stimulatory activity (more than $100 \%$ as compared to the positive control Lipopolysaccharide). The promising novel immunostimulatory activity from this wild mushroom suggests the need for further purification and identification of bioactive molecules which could be used in the treatment of breast cancer.

\section{GPR89A/GPHR is associated with the secretory pathway and is a novel oncogene in breast cancer}

\section{Riccardo Ferro ${ }^{1}$, Pierfrancesco Marra ${ }^{1}$, Sumi Mathew, Alessandra Facchetti, Gianfranco Picco, Patrycja Gazinska, Farzana Noor, Claire Shooter, Priyanka Ghongane, Yanan Zhu, Daniel Weekes, Elodie Noel, Hasan Mirza, Mamun Rashid, Emanuele de Rinaldis, Fara Brasó Maristany, Jelmar Quist, Anita Grigoriadis and Andrew Tutt}

Breast Cancer Now Research Unit, Department of Research Oncology, Guy's Hospital King's College London School of Medicine, London SE1 9RT, UK; ${ }^{1}$ Equal contribution

Aims To characterise the functional role and the suitability as targets for therapy of amplified and overexpressed genes, specifically GPR89A/GPHR, a protein upregulated required for breast cancer cell lines (BCCLs) growth

Procedures Previous analyses of the gene copy-number $(\mathrm{CN})$ alterations and gene expression profiles in a Triple-Negative Breast Cancers
(TNBCs)-enriched cohort of tumour samples identified 141 genes specifically upregulated in tumour with pre-specified features suggesting suitability as drivers of potentially targetable malignant phenotypes. siRNA-based functional validation selected 33 genes specifically required by BCCLs for cell growth. GPR89A/GPHR has been further characterised for its mechanisms of malignant cell-specific addiction.

Major Findings GPR89A/GPHR is amplified and highly expressed in our cohort (60\% of breast cancers overall and $60 \%$ of TNBCs) and in publicly available datasets such as TCGA. GPR89A/GPHR is required for cancer cell proliferation while redundant in non-malignant cells. Published evidence suggests GPR89A/GPHR to be a proton pump controlling the $\mathrm{pH}$ of the Golgi complex. Additional evidence, in plants, suggests high similarity $(65 \%)$ and identity $(45 \%)$ with the Abscisic Acid receptor, a G-protein-coupled receptor, controlling intracellular calcium levels. Our data in BCCLs suggest an Endoplasmic Reticulum (ER) localisation of GPR89A/GPHR, which controls the dynamics/post-translational modification of plasma membrane (PM) proteins such as EGFR, IL15RA and MUC1. GPR89 $\mathrm{KD}$ causes a reduction in the levels of pERK1/2 and an accumulation in the S-phase of the cell cycle.

Conclusion GPR89A/GPHR is upregulated in breast cancer and aberrantly localised to the ER where it controls the Post/translational modification and dynamics of PM proteins and signals through ERK1/ 2 controlling cell cycle and proliferation.

Significance As GPR89A/GHPR is required for proliferation of BCCLs is redundant in non-malignant cells and is potentially druggable as either a GPCR or an ion channel, it shows potential as a suitable target for therapy in breast cancer particularly those with TNBC.

\section{An integrated genomics and RNAi approach identifies the KIFC1 kinesin as a selective target for triple- negative breast cancer with centrosome amplification}

\author{
Nirmesh Patel, Daniel Weekes, Elodie Noel, Konstantinos \\ Drosopoulos, Emanuele de Rinaldis, Mamun Rashid, Hasan \\ Mirza, Fara Brasó-Maristany, Sumi Mathew, Erika Francesch \\ Domenech, Patrycja Gazinska, Farzana Noor, Jelmar Quist, \\ Rebecca Marlow, Anita Grigoriadis, Spiros Linardopoulos, \\ Pierfrancesco Marra, Andrew Tutt
}

Breast Cancer Now Research Unit, Guy's Hospital, King's College London

Background Triple-negative breast cancers (TNBCs) have limited targeted treatment options. Large-scale genomic and transcriptomic studies have advanced our understanding of TNBCs. However, the substantial number of copy number and gene expression alterations in TNBCs makes it difficult to identify putative drivers, biomarkers and/ or therapeutic targets of the disease. To overcome this problem, we have carried out an integrative computational and RNAi-based approach to identify TNBC gene addictions.

Methods Copy number and gene expression alterations in a large breast tumour series enriched for TNBCs were analysed with normal breast controls revealing 141 candidate genes whose upregulated gene expression is copy number driven in TNBC. Of these, RNAi-based functional validation revealed 33 malignant cell-specific addiction genes using an array of breast cancer and non-malignant cell lines. Results STRING analysis of validated hits reveals a subset of genes involved in mitosis. We further validated KIFC1 (HSET) which is known to play a role in clustering supernumerary centrosomes, a common occurrence in breast cancer. We show that siRNA and shRNA-mediated depletion of KIFC1 decreases cell viability and clonogenic ability specifically in centrosome-amplified cell lines, 
which can be rescued upon introduction of an si/shRNA resistant KIFC1. KIFC1 depletion also produces a high level of catastrophic multipolar mitoses specifically in centrosome-amplified cell lines. Furthermore, in vivo studies show that depletion of KIFC1 suppresses tumour growth of centrosome-amplified cell line xenografts.

Discussion We identify and functionally validate novel tumour addiction genes in TNBC. Our data show that KIFC1, a druggable kinesin motor protein, is a promising malignant cell-selective target for therapy expressed in a significant proportion of TNBCs. We validate its cancer-specific role in amplified centrosome clustering showing that KIFC1 plays an essential role in aiding the survival of breast cancer cells that have supernumerary centrosomes both in vitro and in vivo.

\section{Metformin: a new role in breast cancer prevention?}

\author{
Caitriona Tyndall ${ }^{1}$, Marc Gunter ${ }^{2,3}$ \\ and James M. Flanagan ${ }^{1}$
}

${ }^{1}$ Epigenetics Unit, Department of Surgery and Cancer, Imperial College London, UK; ${ }^{2}$ Department of Epidemiology \& Biostatistics, School of Public Health, Imperial College London, UK; ${ }^{3}$ Nutritional Epidemiology Group, International Agency for Research on Cancer (IARC), France

Breast cancer is the most commonly diagnosed cancer in the UK, accounting for $7 \%$ of overall cancer death. Metformin is a biguanide drug used to treat Type II Diabetes Mellitus and has been shown to reduce breast cancer risk by up to twofold in diabetics, particularly in long-term users. Metformin activity leads to metabolic stress, reduced growth signalling and cell death. The mechanisms of metformin action in relation to breast cancer are largely unknown. This study aims to investigate how metformin activity modulates normal breast epithelial cell growth and proliferation to provide insight into the mechanisms by which it may prevent breast cancer. We have conducted a comparison of metformin treatment in 2D and 3D cell culture using two normal breast epithelial cell lines, MCF10A and MCF12A. We have validated metformin activity by RT-qPCR and Western Blot for known targets including AMPK, mTOR and CyclinD1. We used MTT cell viability assays to assess cell survival in response to increasing doses of metformin after 3 and 7 days of treatment. We observed marked differences in response to metformin treatment between the two cell lines, with MCF12A being more resistant in 2D culture with an IC50 of $6 \mathrm{mM}$, compared with an IC50 of $3 \mathrm{mM}$ for MCF10A after 7 days (ANOVA, $p<0.0001$ ). Furthermore, 3D culture indicates increased drug sensitivity and reduced mammosphere formation, suggesting that long-term exposure to metformin is instigating marked changes in normal breast epithelial cell function. In summary, this study has shown that normal breast epithelial cells are sensitive to metformin treatment. Future work aims to investigate the hypothesis that long-term use of metformin may be eliciting stable molecular changes that may persist adding to its protective effects.

\section{Sulforadex targets breast cancer stem-like cells in patient-derived cells and xenograft tumours}

Bruno Simões, D. Alferez, R. Eyre, K. Spence, A. Santiago-Gomez, A. Sarmiento-Castro, I. Tanaka, B. Kohler, D. Howat, S. Howell, R. Clarke

\section{University of Manchester}

Sulforadex (SFX) is a novel therapeutic comprising synthetic sulforaphane (SFN) stabilised within alpha-cyclodextrin. SFN has been studied as an anti-cancer compound for many years; however, its development has hitherto been hampered due to its inherent instability. Breast cancer stem-like cells (CSCs) have been identified in all molecular subtypes and are believed to be the drivers of breast cancer metastasis.

We investigated SFX effects on breast CSC activity using mammosphere formation and aldehyde dehydrogenase (ALDH) activity in patient samples and patient-derived xenograft (PDX) tumours. SFX $(5 \mu \mathrm{M})$ induced a significant reduction in the mammosphere formation efficiency of both primary $(67 \%, \mathrm{n}=8 / 12)$ and metastatic $(80 \%$, $\mathrm{n}=12 / 15$ ) patient cells. Importantly, using a 14-day in vivo 'window' treatment, SFX (300 mg/Kg/day) also abrogated breast CSC activity of early (HBCx34) and metastatic (BB3RC31) PDX tumours.

The majority of breast cancers express the oestrogen receptor (ER) but these hormone-responsive breast cancers frequently develop resistance to endocrine therapies (e.g. tamoxifen). We have established that CSCs in ER+ breast cancer lack ER expression, representing a potential mechanism of therapeutic resistance (Simões et al., Cell Reports, 2015). However, SFX targets such surviving CSCs identified by ALDH positivity and mammosphere-initiating capacity following tamoxifen treatment in vivo in ER+ HBCx34 and BB3RC31 PDX tumours. In ongoing PDX experiments, which comprise endocrine therapy in combination with SFX for a period of 8 weeks, we will test the effects of the combination on tumour regression.

Mechanistically, SFX inhibited the canonical Wnt pathway in MCF-7 cells and their endocrine-resistant derivatives and we are currently exploring SFX activity on other CSC regulatory pathways.

Our data demonstrate the potential of SFX for clinically meaningful improvements to endocrine therapy in ER+ breast cancer by reversing CSC-mediated resistance.

\section{Molecular mediators of mammographic density}

Alastair Ironside, Jenny Gomm, Linda Haywood, Iain Goulding, Jun Wang, Claude Chelala, J. Louise Jones

Centre for Tumour Biology, Barts Cancer Institute, Queen Mary University of London

Introduction Mammographic density (MD), created predominantly by increased stromal tissue, is a major risk factor for the development of breast cancer, though little is known about the biological mechanisms mediating it. Tamoxifen prevents breast cancer in a subset of high-risk women via mechanisms that appear dependent on reduction of MD. Animal models suggest tamoxifen remodels the mammary stroma to a tumour-inhibitory phenotype. This study aims to analyse the effect of tamoxifen on breast fibroblast function and identify pro-tumourigenic pathways contributing to the density-associated risk.

Methods Primary human breast fibroblasts from normal, high-risk or breast cancer patients were treated with hydroxytamoxifen (4OHTam, $100 \mathrm{nM}-5 \mathrm{uM}$ ), the active metabolite of tamoxifen. Fibroblast function was analysed by measuring: proliferation, expression of stromal proteins fibronectin, LOX and collagen 1 ; effects on TGF- $\beta$ signalling via SMAD phosphorylation and upregulation of the myofibroblast marker SMA. Genome-wide analysis was performed using RNA-Seq.

Results Fibroblasts from 25 patients were treated with 4-OHTam. All patients showed reduced proliferation with treatment. $62 \%$ of patients showed reduced fibronectin expression. TGF- $\beta$-mediated upregulation of SMA and fibronectin was consistently inhibited.

RNA-Seq analysis revealed significant downregulation of Wnt signalling, an established pro-fibrogenic and pro-tumourigenic pathway, and modulation of many metabolic pathways, including components 
of the microsomal anti-oestrogen binding site (AEBS). Binding of tamoxifen to the AEBS inhibits $\mathrm{ChEH}$ activity promoting an antitumourigenic phenotype. The effects of tamoxifen on fibroblasts could be replicated using tesmilifene, a commercially available inhibitor of ChEH.

Conclusion These data indicate that tamoxifen can directly remodel the stromal microenvironment, generating a less 'reactive' stroma. Thus, tamoxifen impacts on multiple pathways, many independent of the oestrogen receptor, to create a tumour-inhibitory microenvironment. This offers exciting potential for patient monitoring and alternative breast cancer prevention strategies.

\section{The effect of cyclopamine on hedgehog signalling pathway in breast cancer cells}

\section{Arwa Flemban, David Qualtrough \\ University of The West of England, Umm Al-Qura University}

Introduction Breast cancer in the second leading cause of death in women worldwide, with a risk of 1 in 8 women developing this disease at some point in their lives. Breast cancer is also the most diagnosed cancer in females. Most deaths associated with this illness are caused by metastasis. Cancer metastasis involves the process of epithelial-mesenchymal transition (EMT) and understanding this process and its regulatory mechanism in breast cancer could propose answers of the disease prognosis, thereby identifying possible targets for therapy. Hedgehog (Hh) signalling pathways were found to be involved in in vivo and in vitro studies of human cancers. It is suggested that misappropriation of Hh pathway is involved in breast neoplasia and cancer stem cell and breast tumour cell metastases. Yet, the involvement of this pathway in breast cancer subtypes remains poorly understood.

Aims The current study aimed to investigate the effect of cyclopamine, a plant-derived alkaloid inhibitor of $\mathrm{Hh}$, on cellular yield, apoptosis, EMT, and Hh markers' expression (E-cadherin, $\beta$-catenin, Gli1, Gli2, and Gli3). Moreover, localisation of these markers was also assessed in breast cell lines.

Procedures Breast cancer cell lines MCF7, MDA-MB361 (Luminal), BT20, MDA-MB-231 (Basal and Triple-negative), MDA-MB-453, and SKBR3 (HER2+ ve) were used in this study. In addition, normal breast cell line (MCF10A) was also included. These cells were treated with three concentrations of cyclopamine $(1,5$, and $10 \mathrm{M})$ for $48 \mathrm{~h}$ and compared with untreated cells. Cell apoptosis was evaluated by Annexin V kit. Moreover, the expression of E-cadherin, $\beta$-catenin, Gli1, Gli2, and Gli3 in these cells was assessed by immuno-cytofluorescence and flow cytometry.

Major findings Cyclopamine reduced cell number and induced cell apoptosis in a dose-dependent manner; this reduction was depending on the type of breast cancer cell line. Additionally, cyclopamine decreased the expression of Gli and changed its nuclear localisation. E-cadherin expression increased in some of the breast cancer cells after treatment, and there was a reduction in the level of $\beta$-catenin expression.

Significance of the research and Conclusions The present study demonstrated that the expression of Gli markers varied according to the type of breast cancer and the level of expression and localization of Gli changed after treatment with cyclopamine. The data indicated Hh signalling pathway involved in breast neoplasia and the misappropriation in $\mathrm{Hh}$ signalling differed according to the subtype of breast cancer. That also indicated that cancerous cells response to cyclopamine treatment varies according to subtypes.
Evaluating the Estimated Lifetime Oestrogen Exposure (ELEE) model as a risk factor for breast cancer

\author{
Annelie Johansson ${ }^{1}$, EPIC-Italy Investigators, Paolo Vineis ${ }^{2,3}$, \\ and James M. Flanagan ${ }^{1}$
}

${ }^{1}$ Epigenetics Unit, Department of Surgery and Cancer, Imperial College London, UK; ${ }^{2} \mathrm{MRC} / \mathrm{PHE}$ Centre for Environment and Health, School of Public Health, Imperial College London, UK; ${ }^{3}$ Human Genetics Foundation, Torino, Italy

It is well established that oestrogen levels are involved in the aetiology of breast cancer. Numerous hormonal risk factors have been separately well studied and shown to increase risk, including early menarche, late menopause, nulliparity, later age at first fullterm pregnancy, oral contraceptives, hormone replacement therapy, and increased BMI for postmenopausal women. How these factors interact and combine to affect breast cancer risk, however, has been less well characterised. We aimed to develop a mathematical model to estimate a woman's lifetime exposure to oestrogen based on hormonal risk factors. We used a case-control study design nested within the European Prospective Investigation into Cancer and Nutrition (EPIC) Italy cohort $(\mathrm{n}=710$ postmenopausal breast cancer cases matched with 2 controls per case, $n=1420$ ). For each hormonal risk factor, the odds ratio (OR) was estimated using conditional logistic regression analysis. The first Estimated Lifetime Oestrogen Exposure (ELEE) model was calculated simply as the reproductive time between menarche and menopause. We observed a $12 \%$ increased breast cancer risk for each additional year to a woman's reproductive time $(\mathrm{OR}=1.12,95 \%$ Confidential Interval (CI): $1.00-1.25)$. Parity shortens the reproductive time and was subtracted from the reproductive time in the second ELEE model, and the $\mathrm{z}$-score of BMI was added since it showed a strong association with increased breast cancer risk $(\mathrm{OR}=1.04,95 \% \mathrm{CI}$ : 1.02-1.06) within the dataset. This modified ELEE model was further associated with breast cancer risk $(\mathrm{OR}=1.14,95 \% \mathrm{CI}$ : 1.06-1.22). The introduced ELEE models support the association between oestrogen exposure and increased risk for breast cancer, and we conclude that the reproductive time, parity, and BMI in combination are a valid approximation for a woman's total oestrogen exposure. Future studies will focus on developing the ELEE model in premenopausal women and introduce other hormonal and lifestyle risk factors.

\section{Developing a decision aid around genetic testing for young women with breast cancer-the YoDA BRCA Study}

Grimmett C. ${ }^{1}$, Brooks C. ${ }^{1}$, Recio-Saucedo A. ${ }^{1}$, Cutress R. ${ }^{2}$, Copson E. ${ }^{2}$, Evans G.D. ${ }^{3}$, Gerty S. ${ }^{2}$, Armstrong A. ${ }^{4}$, Turner L. 5 , Mason S. ${ }^{5}$, Ahmed M. ${ }^{6}$, Eccles B. ${ }^{2}$, Eccles D. ${ }^{7}$, and Foster C. ${ }^{1}$

${ }^{1}$ Macmillan Survivorship Research Group, Faculty of Health Sciences, University of Southampton, Southampton, UK; ${ }^{2}$ Cancer Sciences, University of Southampton, Southampton, UK; ${ }^{3}$ Genetic Medicine, University of Manchester, UK; ${ }^{4}$ Christie Hospital NHS Trust, Manchester, UK; ${ }^{5}$ Patient and Public Involvement; ${ }^{6}$ Wessex Clinical Genetic Service, Southampton, UK; ${ }^{7}$ University

of Southampton, Clinical Trials Unit, UK 
Background Younger women diagnosed with breast cancer are more likely to have inherited genetic mutations. Genetic testing around the time of diagnosis is becoming more common with genetic testing being increasingly offered to inform treatment decisions. Information to support decision making for young women is often unavailable outside of specialist genetics services. Decision aids (DA) can support women to make informed choices.

Aim To explore the views and experiences of young women about genetic testing and their information preferences to inform the development of a web-based DA.

Methods Participants were $\leq 50$ years and $\leq 18$ months of diagnosis and recruited from two NHS hospitals. Purposive sampling ensured inclusion of women with a range of characteristics relating to genetic testing. Semi-structured interviews and focus groups were employed. Verbatim transcripts were interrogated using a framework approach. Results 29 women participated in interviews; 15/29 women had undergone genetic testing ( $\mathrm{N}=3$ BRCA-positive results). The following themes emerged as important for decision making regarding genetic testing: personal attitudes/values regarding genetic testing; knowledge of reasons to have/not have testing, test processes and implications of testing for themselves and family members. Clear 'jargon-free' information was desired and level of information preferences varied within and between individuals. Two focus groups $(\mathrm{N}=7$ in each) highlighted the importance of a summary of each area of interest, followed by further information, warnings prior to accessing sensitive information and avoiding terms such as 'faulty' gene'.

Conclusion Findings from the interviews and focus groups will be used to develop a prototype DA to be refined in think-aloud interviews. The final DA will endeavour to support decision making regarding whether or not to have genetic testing. Future research will include testing the finalised DA in a randomised controlled trial.

\section{Defects in ARID1A and other BAF complex tumour supressor genes sensitise tumour cells to clinical inhibitors of the DNA damage checkpoint kinase, ATR}

\section{Chris Williamson}

\section{The Institute of Cancer Research}

Identifying genetic biomarkers of synthetic lethal drug sensitivity effects provides one approach to the development of precision medicine treatments for cancer. Mutations in BAF complex tumour suppressor genes represent one of the most common molecular alterations in human cancer. However, therapeutic approaches that selectively target BAF defects are not yet clinically available. Here we demonstrate that defects in ARID1A and other BAF complex tumour suppressor genes sensitise tumour cells to clinical inhibitors of the DNA damage checkpoint kinase, ATR, both in vitro and in vivo. Mechanistically, ARID1A deficiency results in topoisomerase 2A and DNA decatenation defects, which cause an increased reliance on ATR checkpoint activity. In ARID1A mutant tumour cells, inhibition of ATR triggers premature mitotic entry, genomic instability and apoptosis. ATR inhibitors are currently in Phase I clinical trails as combination therapies. The data we present here provides the preclinical and mechanistic rationale for assessing BAF defects as biomarkers of single-agent ATR inhibitor response and represents a novel synthetic lethal approach to targeting tumour cells.

\section{Bisphenol A alternatives can effectively substitute for estradiol in promoting cell growth through estrogen receptors in human breast cancer cells}

\author{
Robin Mesnage, Alexia Phedonos and Michael \\ N. Antoniou
}

Gene Expression and Therapy Group, King's College London, Faculty of Life Sciences \& Medicine, Department of Medical and Molecular Genetics, Guy's Hospital London

Background Plasticizers with estrogenic activity, such as bisphenol A (BPA), have been reported to have potential adverse health effects in humans, especially in fetal and infant stages. Due to mounting evidence and public pressure, BPA is being phased out by the plastics manufacturing industry and is being replaced by other bisphenol variants in "BPA-free" products. We have compared estrogenic activity of 7 bisphenol analogs (BPA; bisphenol S, BPS; bisphenol F, BPF; bisphenol AP, BPAP; bisphenol AF, BPAF; bisphenol Z, BPZ; bisphenol B, BPB).

Methods Activation of estrogen receptors was determined using a mammary T47D cell line harboring a stably integrated copy of a luciferase reporter gene under control of a promoter containing estrogen response elements. Cell proliferation induced by the activation of estrogen receptors was determined in three breast cancer cell lines (MCF-7, T47D, and MDA-MB-231) by the conventionally used E-screen assay. The natural hormone estradiol was used as a reference.

Results All bisphenols were able to substitute for the natural hormone in promoting cell growth through estrogen receptors. In both assays, BPAF was most potent bisphenol (50\% effective concentration for receptor activation $=0.08 \mu \mathrm{M})$ followed by $\mathrm{BPB}(0.3 \mu \mathrm{M})<\mathrm{BPZ}$ $(0.4 \mu \mathrm{M}) \sim \operatorname{BPA}(0.4 \mu \mathrm{M})<$ BPF $(1 \mu \mathrm{M}) \sim \operatorname{BPAP}(1 \mu \mathrm{M})<$ BPS $(3 \mu \mathrm{M})$. The addition of ICI $182,780(100 \mathrm{nM})$ antagonized the activation of estrogen receptors by both estradiol and bisphenols.

Conclusions Our results show that BPA-free products are not necessarily safer. Three bisphenols (BPAF, BPB, BPZ) were more estrogenic than BPA. The clinical relevance of human exposure to BPA alternatives in hormone-dependent breast cancer progression should be investigated.

\section{Restricted glycolysis promotes stem cell-like characteristics in breast cancer cells}

Arindam Banerjee, P. Arvinrad, M. Darley, Stephanie Laversin, Ramsey Cutress, Stephen Beers, Franchesca Houghton, Charlie Birts and Jeremy Blaydes

Cancer Sciences, Faculty of Medicine, University of Southampton, United Kingdom

Increased glucose uptake is a characteristic feature of many cancer cells, including those from breast tumours. However, during cancer progression and metastasis, cancer cells can modulate their glucose utilisation to overcome localised nutritional limitations in hostile microenvironments. We hypothesised that this plasticity in glucose 
utilisation could result in changes in the cells' oncogenic potential. To investigate this, we established a panel of human breast cancer cell lines containing matched pairs with either standard (i.e. high) or reduced rates of glycolysis. Glycolysis was reduced by adapting cells to grow in $10 \mathrm{mM}$ fructose, compared to the standard $25 \mathrm{mM}$ glucose. Next, we investigated the cells' oncogenic potential by various cell function-based studies (3D matrigel assay and cell invasion assay) in in vitro conditions. Furthermore, we also determined whether glycolytic rates influence any changes in the cancer stem cell-like (CSC) population by specifically identifying their mammosphere- forming ability and changes in the CD44high/CD24low/-ve and ALDH1 + ve cell populations. Significant increases in growth in 3D matrigel, cell invasion, and the proportion of cells with CSC-like properties were observed across all the glycolytically restricted cells, compared to their glycolytic counterparts. Hence, metabolic adaptation of breast cancer cells to glucose-restricted conditions results in more invasive and stem cell-like features. Molecular identification of key molecules involved in this process is ongoing, as we anticipate that they may include potential prognostic biomarkers and/or therapeutic targets.

\section{Modelling MCL-1 inhibition in breast cancer}

\author{
Kirsteen Campbell, Sandeep Dhayade, Nicola Ferrari, Emma \\ Johnson, Jane Hair, Joanne Edwards, Stephen Tait and Karen \\ Blyth
}

\section{Beatson Institute}

Aims To test the hypothesis that MCL-1 is required for both the development and maintenance of breast cancer and to investigate the therapeutic potential of MCL-1 inhibition in breast cancer.

Procedures Approaches used include in silico analysis of MCL-1 expression in publicly available datasets and immunohistochemistry on breast tumour tissue microarrays. Small-molecule MCL-1 inhibitors and knock-down/knock-out of MCL-1 in in vitro and in vivo models were also used to investigate the therapeutic potential of targeting MCL-1 in breast cancer.

Major findings We find that MCL-1 is frequently elevated in breast cancer. Strikingly, this is most prominent in the basal subtype, regarded as the most stem cell-like of the molecular classifications that is both highly aggressive and has limited therapeutic options. Moreover, we find high expression of MCL-1 protein in human breast cancer tissue samples, especially in ER-negative disease, and find associations between high MCL-1 and poor prognosis. We show that inhibition or knock-down of MCL-1 in breast cancer cell lines in vitro and in vivo impairs tumour growth, and we are now using GEM models to further investigate the role of MCL-1 in breast cancer development and response to therapy.

Significance of research Inhibition of anti-apoptotic BCL-2 function, using drugs called BH3-mimetics, shows considerable promise in the treatment of haematological malignancies. Drugs specifically targeting the BCL-2 family member MCL-1 are under development, and our data suggest that these will have the potential to improve the treatment of breast cancer.

Conclusions Elevated MCL-1 is associated with poor prognosis in breast cancer and MCL-1 inhibition may provide a new therapeutic approach.

\section{Characterisation of a new mechanism of ATR/Chk1 regulation in breast cancer: role of $\mathrm{mTORC2}$}

\author{
Jogitha Selvarajah, Veronica A. Carroll
}

St George's, University of London

mTOR is a serine-threonine kinase belonging to the PIKK family and acts as a central node to integrate signalling from growth and stress factors to regulate cell growth. Other members of the PIKK family are ATM, ATR and DNA-PKcs, which all have established roles in the DNA damage response. mTOR is the catalytic component of functionally distinct complexes mTORC1 and mTORC2. Recent data show that mTORC2 complex also has tyrosine kinase activity. Dysregulation of mTOR signalling occurs in numerous cancers, particularly in breast cancer, leading to the use of mTORC1 inhibitors such as the rapamycin analogue (rapalog), everolimus, for advanced hormone receptor-positive, HER2-negative breast cancer in combination with exemestane. There are many ongoing clinical trials of rapalogs in combination therapy including DNA-damaging agents to treat breast cancer as well as mTOR kinase inhibitors that target both mTORC1 and mTORC2 complexes. Using breast cancer cell lines and biochemical methods, we have recently demonstrated that there is significant crosstalk between the nutrient and stress sensing mTOR pathway and the DNA damage response activation of ATR/Chk1. We recently demonstrated that DNA damage-induced cell cycle arrest at $\mathrm{S}$ and G2/M phase is dependent on mTOR. Specifically, mTORC2 was required for the activation of Chk1, a key cell cycle regulator, at the level of Chk1 protein production. Furthermore, in a panel of breast cancer cell lines, with varying degrees of chemoresistance, an mTOR kinase inhibitor was able to overcome resistance to DNA-damaging agents by potentially destabilising cell cycle arrest and thereby enhancing DNA damage-induced cell death. These data suggest that mTORC2-Chk1 signalling is a survival pathway in breast cancer which has important implications for breast cancer combination therapy.

\section{Functional investigation of a putative breast cancer risk variant}

Joe Baxter, Nicola H. Dryden, Laura R. Broome, Nichola Johnson, Sarah L. Maguire, Nick Orr, Olivia Fletcher

The Institute of Cancer Research, London

Two independent tagging SNPs (rs13387042, rs16857609), mapping to $2 \mathrm{q} 35$, have been strongly associated with increased breast cancer susceptibility. For one of these (rs13387042), fine-mapping and functional studies have identified a putative causal variant which influences FOXA1 binding to a transcriptional enhancer of IGFBP5. To identify a second putative causal variant, we made the following assumptions: (i) this causal variant would be correlated with rs 16857609 , (ii) it would be associated with levels of expression of IGFBP5, and (iii) it would physically interact with IGFBP5 to influence expression.

We identified 106 SNPs that were correlated $(\mathrm{r} 2>0.2)$ with both rs16857609 and an IGFBP5 eQTL SNP (rs6716223). Of these, 8 also mapped to a region within the $2 \mathrm{q} 35$ locus that formed long-range $(>700 \mathrm{~kb}$ ) physical interactions with the IGFBP5 promoter, based on 
Capture Hi-C interaction data. Consulting breast-relevant ChIP-seq (FOXA1, GATA3, ER- $\alpha$ ) and DNase-seq data, we were able to identify a single SNP that maps to a region exhibiting characteristics of a functional element-rs78040234.

Regulatory properties of the region were first assessed by luciferase reporter assay. In the context of the IGFBP5 promoter, the region containing rs78040234 significantly upregulated the expression of the reporter $(\mathrm{p}<0.001)$; moreover, a single-nucleotide substitution of rs78040234 to the minor, risk-associated allele reduced the upregulatory activity $(\mathrm{p}=0.02)$. We are currently generating breast cancer cell lines with deletions and substitutions in the rs78040234 region to investigate the functional element and the role of the SNP in an endogenous context.

\section{Translocation-induced gene breakage identifies recurrent fusion gene candidates}

\author{
Aisyah Mohd Noor' ${ }^{1}$, Sarah Maguire ${ }^{2}$, Johnathan Watkins ${ }^{1}$, \\ Jelmar Quist $^{1}$, Hasan Mirza', Kayleigh Ougham ${ }^{1}$, Andrew Tutt ${ }^{1}$, \\ Cheryl Gillett ${ }^{3}$, Rachael Natrajan ${ }^{2}$, Anita Grigoriadis ${ }^{1}$
}

${ }^{1}$ Breast Cancer Now Research Unit, Guy's Hospital, King's College London, London SE1 9RT, UK; ${ }^{2}$ Breast Cancer Now Research Centre, Institute of Cancer Research, London SW3 6JB UK; ${ }^{3}$ King's Health Partners Cancer Biobank; Research Oncology, Guy's Hospital, King's College London, London SE1 9RT, UK

Breast cancer is characterised by widespread structural and chromosomal rearrangements and fusion genes that influence transcription, leading to aborted transcription, gene inactivation or gene activation. We aimed to identify disrupted transcripts with underlying genomic alterations and characterise their features in breast cancers.

Affymetrix SNP6.0 and Exon1.0ST data of 123 in-house primary breast tumours were analysed with a walking Student's $t$ test to identify disrupted transcripts with proximal genomic breakpoints. SNP6, exonarray and RNA-seq data of 51 breast cancer cell lines (BCCL) and 152 BRCA samples from The Cancer Genome Atlas (TCGA) were used for validation. Fusion transcripts were detected using the ChimeraScan algorithm. Samples were molecularly characterised by immunohistochemistry for hormone receptors (ER and PR), PAM50, IntClust and TNBC subtyping, genomic instability measures, and gene signatures.

We identified 106 out of 877 disrupted transcripts with proximal genomic breakpoints, and recurrent in at least five samples. RNA-seq of BCCL and TCGA data depicted 33/106 as gene fusion partners. Thirtyone transcripts show putative oncogenic roles in literature and 26 were found as potential fusion partners in breast cancers. While 20 transcripts had diverse fusion partners, 13 transcripts consistently fused to unique partners (range $=2-31$, median $=3$ ). Three novel fusions were recurrent across all datasets with multiple junction-spanning and uniquely aligned reads (CDK14:CLDN12, $n=6 ;$ FOXP1:EIF4E3, $n=10$; lncRNA BC090059:RIN2, $n=89$ ). IHC receptor subtype specificity was observed for $7 / 33$ aborted transcripts. Aborted transcription of CDK14 and FRMD5 was more prevalent in PAM50-Luminal A and HER2 samples, respectively. Overall aborted transcription was less prevalent in the TNBC-LAR subtype (Fisher's $p$ value: 0.034 ).

We show that integration of exon expression and copy number data can identify genes with disruption in their transcriptional units, some of which represent fusion genes. Characterisation of these transcripts, their partners and their prevalence with certain molecular features can provide further knowledge to their biological significance in breast cancers.

\section{Identification of HORMAD1-induced synthetic sensitivities in triple negative breast cancers}

\section{Dalia Tarantino, Dan Weekes, Helen Pemberton, Jessica Frankum, Chris Lord, Jelmar Quist, Anita Grigoriadis, Andrew Tutt}

Breast Cancer Now Research Unit, Guy's Hospital, King's College London

Triple-Negative Breast Cancers (TNBCs) are characterised by high levels of genomic instability driven by DNA repair deficiencies. HORMAD1, normally only expressed in germline cells, was found to be present in $\sim 60 \%$ of TNBCs and can induce genomic instability through inhibition of DNA repair by Homologous Recombination. The genes and pathways modulated in response to HORMAD1 expression in mitotic cells are currently unknown, but represent potentially targetable synthetic sensitivities in TNBC.

To identify such pathways, an isogenic inducible HORMAD1expressing triple-negative breast cancer cell line SUM159 clone and its parental isogenic control clone were screened with an RNA interference library consisting of pools of 4 oligos targeting 1743 genes, including kinases, oncogenes, tumour suppressors and epigenetic-related genes. Cell viability was evaluated with CellTiter-Glo. Sensitising hits were validated in a secondary deconvolution screen and in two GFP-expressing SUM159 clones to exclude sensitivities caused by ectopic protein expression alone.

Our siRNA screen identified 63 genes which induced high levels of sensitivity upon HORMAD1 expression and showed an enrichment in genes involved in DNA repair. Out of those, 14 were tested and eight genes were validated as having an on-target effect. Furthermore, for 7 of these genes, namely XRCC1, TDP1, DCLRE1A, POLH, NTHL1, BRIP1 and ATR, HORMAD1-induced sensitivity was confirmed in 2 additional HORMAD1-inducible expression clones.

Our data indicate that pathways that prevent replication fork stalling or involved in their restart such as base excision repair (XRCC1), DNA interstrand cross-link repair (DCLRE1A, FANCC) and translesion synthesis $(P O L H)$ are involved in the tolerance of HORMAD1 expression in mitotic cells. These compensatory DNA repair mechanisms represent potentially targetable pathways in HORMAD1-expressing TNBC and warrant further exploration.

\section{Dissecting cancer cell metabolism in a 3D breast model}

\section{Caroline Barker, Papadakis, E.S., Cutress, R., Blaydes, J.P}

University of Southampton

Glycolysis and fatty acid oxidation are being targeted therapeutically in cancer, as these metabolic pathways are upregulated in cancer cells. The breast can be modelled in 3D using an MCF-10A acini model, with oncogene overexpression (MCF-10A:HER2) altering acinar morphogenesis to mimic DCIS, a breast cancer precursor. We have published these models and their relevance for the study of pharmacological inhibition; here we specifically examine the effect of molecular and pharmacological alteration of metabolism on MCF-10A morphogenesis.

To disrupt the cellular metabolic sensor machinery, pooled populations overexpressing $\mathrm{CtBP} 2$ were generated by retroviral transduction. To study the sensitivity of our models to therapeutic agents, acini were cultured in 3D for 12 days, with metabolic 
inhibitors present from day 6. Acinar morphogenesis was studied using confocal microscopy. 2D cell viability was assessed by ATP and Alamar blue assays. All results are from three independent experiments each containing three technical replicates.

Overexpression of the metabolic sensor CtBP2 interferes with acinar morphogenesis $(p \leq 0.001)$, promoting transformation. This is reversible by $\mathrm{CtBP}$ inhibition. The metabolic inhibitors etomoxir $(p \leq 0.001)$ and oxamate $(p \leq 0.01)$ reversed HER2-driven transformation of acini. Dichloroacetate had no effect on MCF10A:HER2 acini but strikingly drove controls towards a transformed phenotype $(p \leq 0.001)$. In $2 \mathrm{D}$ cultures, oxamate inhibited viability $(\mathrm{p} \leq 0.01)$ while etomoxir and dichloroacetate did not.

Manipulation of metabolism altered the transformation of MCF-10A acini, highlighting the relevance of metabolism in cancer. The identification of a phenotype in 3D that was not observed in 2D reinforces the importance of 3D culture systems to examine pharmacological agents.

\section{The role of TP53 in determining breast tumour subtype}

Kate Packwood, M. Sommerlad, G. T. Martland,

R. Pengelly, E. C. Shaw, J. P. Blaydes, J.C. Strefford,

G. J. Thomas, A. C. Bateman, D. M. Eccles

\section{University of Southampton}

Background A germline TP53 mutation predisposes to young breast cancer and other tumours recognized clinically as Li-Fraumeni Syndrome (LFS). We hypothesized that an inherited TP53 mutation is important in driving a specific breast tumour subtype and that timing of TP53 malfunction may be relevant to subtype.

Methods We collected 136 breast cancer formalin-fixed paraffin-embedded (FFPE) blocks from TP53 mutation carriers. Morphological review was performed by two independent pathologists. Tissue microarrays were constructed from ductal carcinoma in situ (DCIS) and tumour tissue. A 48-sample HaloPlex-targeted enrichment kit was used to characterise the genetic landscape and investigate timing of TP53 loss. Results Tumours were typically high-grade ductal tumours of no special type, associated with widespread high-grade DCIS. HER2 overexpression was found in $63 \%$ of cases compared with $24 \%$ of an age-matched cohort (Copson et al. J Natl Cancer Inst. 2013 105(13):978-88.) There was a high frequency of tumours containing a sclerotic, collagen-rich stroma $(85 \%$ of cases) and a similar proportion stained positive for stromal $\alpha$-smooth muscle actin, indicating myofibroblast differentiation and suggesting activation of TGF- $\beta 1$ signalling. Moderate to strong tumour staining for integrin $\alpha v \beta 6$ (an activator of latent TGF- $\beta 1$ ) was present in $50 \%$ of cases. Targeted Next-Generation Sequencing (NGS) of tumour DNA confirmed the germline mutation across cases, with a suggestive loss of heterozygosity ( $\mathrm{LOH})$ of the wild-type TP53 allele. Somatic mutations in cancer genes including PIK3CA, NOTCH1, ATM, ATR, AKT1 and ERBB2 were detected. TP53 status will be confirmed in the matched DCIS precursor lesion.

Conclusion This supports the hypothesis that a germline TP53 mutation strongly predisposes to HER2-amplified, high-grade tumour subtype. TGF $\beta$ signalling and LOH of WT TP53 are likely drivers in LFS breast tumours. NGS is successful in identification of somatic mutations in archival FFPE material.

\section{Combining patient-matched diagnostic and on-treatment biomarkers significantly improves prediction of response to endocrine therapy}

Andrew Sims, Arran Turnbull, Dominic Pearce, Yan Ping Lee, Lorna Renshaw, Sonya Uddin, Anu Fernando, Hannah Webb, Jeremy Thomas, Mike Dixon
University of Edinburgh

Background Identifying which breast cancer patients will recur on endocrine therapy is a major challenge. Many multigene classifiers are available for predicting prognosis. These are used at diagnosis to determine whether adjuvant endocrine treatment alone is sufficient for $\mathrm{ER}+$ patients to remain disease free, or if additional therapy is required. We developed a test measuring diagnostic IL6ST and a 2-week proliferation marker (either at transcript level or by immunohistochemistry, which accurately predicts neoadjuvant response (NR), recurrence-free survival (RFS) and breast cancerspecific survival (BCS).

Methods We compared predictions using our test with established clinicopathological markers or estimations of multigene classifier scores (Oncotype DX, EndoPredict, Prosigna, MammaPrint) before and/or on treatment with aromatase inhibitors (AIs). We analysed diagnostic and 2 week biopsies from 3 patient cohorts: A: $n=73$, treated in Edinburgh with neoadjuvant (3 months) then adjuvant letrozole, B: $n=44$, treated at Royal Marsden with neoadjuvant anastrozole ( 3 months), and C: $n=84$, receiving 2 weeks of AIs prior to surgery and in the adjuvant setting. NR was determined using ultrasound and cohorts A and C had 10 years follow-up.

Results Prediction accuracy of NR at 3 months was poor (30-70 \%) with existing methods using either pre or on treatment measures alone. The most accurate predictions all utilised IL6ST level at diagnosis and a proliferation marker (MCM4 or Ki67) on treatment (70-96\%). Improvements were also observed with outcome prediction: $\mathrm{p}=0.0005$ (RFS) and $\mathrm{p}=0.0002$ (BCS) in series $\mathrm{A}$ and $\mathrm{p}<0.0001$ for both RFS and BCS in series C. Hazard ratios calculated using our test were significantly higher than with all other methods tested either pre or on treatment.

Conclusions Measuring IL6ST at diagnosis and proliferation at 2 weeks outperforms all current predictive classifiers and clinical tools for both short-term (NR) and long-term (RFS and BCS) outcomes for AI therapy.

\section{A 3D in vitro model of the human breast duct: unravelling myoepithelial-luminal interactions in breast cancer}

\section{Edward Carter, J.J. Gomm, L.J. Jones, and R.P. Grose}

\section{Barts Cancer Institute}

The ducts of the human breast are composed of an inner layer of luminal cells supported by an outer layer of myoepithelial cells. This bilayer is critical for the normal function of the breast and breaks down in cancer. During DCIS, once luminal cells have adopted a cancerous disposition, the myoepithelial layer remains intact but is subsequently lost in invasive cancer. Interrogating the myoepithelialluminal relationship in a physiomimetic setting is therefore critical for understanding how breast cancers are able to progress from DCIS to invasive cancer. However, currently no system exists which models this bilayer structure in a manner that can be easily manipulated by researchers. We have therefore developed such a model, using primary tissue obtained through the Breast Cancer Now tissue bank.

Populations of myoepithelial and luminal cells were isolated from reduction mammoplasty specimens based on their expression of CD10 and EPCAM. These pure populations of cells were cultured separately without losing their respective differentiated phenotype and were susceptible to lentiviral infection, allowing genetic manipulation of either cell type while in $2 \mathrm{D}$ culture. 
Fluorescent labelling of these cells allowed the tracking of their behaviour once placed into a 3D environment. Once recombined, these two cell types coalesced to form spherical and elongated ductlike structures. These structures comprised a layer of luminal cells surrounded by myoepithelial cells. Confocal imaging of myoepithelial and luminal markers revealed reconstitution of a bilayer structure absolutely reflective of normal physiology.

This novel 3D model of the human breast duct presents a powerful tool with which to dissect myoepithelial-luminal interactions in the early stages of breast cancer, and will help uncover markers to predict DCIS patients who will progress towards invasive breast cancer.

\section{A comparative study of male versus female breast cancer identifies overexpression of eiF signalling pathways in male breast cancer providing opportunities for a therapeutic window}

\section{Matthew Humphries, Charlotte Suleman, Andrew Hanby and Valerie Speirs on behalf of the Male Breast Cancer Consortium}

Leeds Institute of Cancer and Pathology, University of Leeds

Background Although rare, male breast cancer (MBC) is becoming more common, yet remains understudied. Treatments are informed by clinical studies conducted in women, based on assumptions that underlying biology is the same, although evidence suggests this may not be the case.

Methods A case-matched transcriptomic investigation of MBC and female breast cancer was performed. Transcriptomic data was confirmed by qRT-PCR and biomarkers assessed immunohistochemically on $477 \mathrm{MBC}$ samples represented on tissue microarrays and related to survival. MicroRNA (miR) expression was determined in a subset of cases by RNA-seq.

Results Hierarchical clustering and Pathway Ingenuity Analysis identified gender-specific gene expression patterns. Expression of specific eIF transcripts was up-regulated in MBC, confirmed by qRTPCR. By immunohistochemistry, eIF4E and eIF5 were negatively prognostic for overall survival $(\mathrm{p}=0.012 ; \mathrm{HR}=1.77,1.12-2.8$ and $\mathrm{p}=0.033 ; \mathrm{HR}=1.68,1.04-2.74$, respectively). Effects on overall survival remained independently prognostic upon multivariate analysis (eIF4E $\mathrm{p}=0.05$; HR 2.13 (1.0-4.6), eIF5 $\mathrm{p}=0.04$; HR 2.63 (1.04-6.65), respectively). Co-expression of eIF4E and -5 $(\mathrm{p}=0.005 ; \mathrm{HR}=2.471,1.280-4.770)$ also impacted on survival, remaining so upon multivariate analysis $(\mathrm{p}=0.002$; HR 6.205, 1.99-19.30). miR analyses on a subset of MBC cases revealed expression of 19 miRs which may regulate eIFs.

Conclusions Our findings support the notion that breast cancer is different between genders. Overexpression of eIF4E and eIF5 suggests these proteins could represent predictive biomarkers in MBC. With mTOR inhibitors which target this pathway now in the clinic, these biomarkers may represent new targets for therapeutic intervention, although further independent validation is required.

\section{P-glycoprotein (ABCB1) expression during breast cancer development is regulated by HuR-dependent loading of miR-19b at non-canonical sites}

James L. Thorne ${ }^{1}$, Sebastiano Battaglia ${ }^{2}$, Samir S. Jana ${ }^{3}$, Rebecca A. Millican-Slater ${ }^{4}$, Laura Smith ${ }^{5}$, Laura Wastall ${ }^{5}$ and Thomas A. Hughes 5
${ }^{1}$ School of Food Science and Nutrition, University of Leeds, UK; ${ }^{2}$ Center for Immunotherapy, Roswell Park Cancer Institute, Buffalo, USA; ${ }^{3}$ University of Calcutta, Kolkata, India; ${ }^{4}$ Leeds Teaching Hospitals NHS Trust, Leeds, UK; ${ }^{5}$ School of Medicine, University of Leeds, UK

Chemotherapy resistance remains a major barrier to successful breast cancer (BC) treatment. Therapies that inhibit drug efflux pumps to overcome resistance have failed due to off-target side effects. Manipulating tumour-specific pathways of drug efflux pump expression could target chemoresistance, whilst simultaneously overcoming side effects. Our aim was to examine BC-specific, post-transcriptional regulation of $\mathrm{P}$-glycoprotein $(\mathrm{ABCB} 1)$, an efflux pump long associated with chemoresistance.

First, we determined the ABCB1 untranslated regions (UTRs) expressed in BC and established UTRs previously implicated as regulatory were broadly undetectable in primary $\mathrm{BC}$ samples or cell lines. Next, we screened for potential microRNA regulators by in silico prediction of binding to the expressed ABCB1 $3^{\prime}$ UTR. The candidate pool was refined using a $\mathrm{BC}$ cohort of 302 patients by eliminating microRNAs not expressed or without an inverse correlation to ABCB1 mRNA. We further screened by examining microRNA (qPCR) and P-glycoprotein (IHC) expression across a panel of matched normal breast, DCIS and invasive BC tissues. P-glycoprotein was significantly up-regulated across this progression, and one microRNA, miR-19b, was significantly both down-regulated and negatively correlated with P-glycoprotein, identifying it as the single candidate regulator.

Subsequent biochemical analyses found exogenous miR-19b overexpression reduced P-glycoprotein protein expression and enhanced intracellular epirubicin retention and associated cell death. Surprisingly, mutation of the predicted miR-19b seed region did not abrogate miR-19b-dependent regulation. In fact, sequential deletion of the UTR identified a miR-19b-responsive region devoid of a miR$19 \mathrm{~b}$ seed site. In silico analysis revealed a consensus sequence for the RNA-binding factor HuR. Both activity and expression of HuR were essential for miR-19b loading to this non-canonical site.

Although miR-19b and HuR have been reported as putative oncogenes, their role in breast cancer appears more equivocal and targeting either could lead to chemoresistance.

\section{Identifying molecular drivers and early diagnostic biomarkers in breast cancer leptomeningeal metastasis by the interrogation of cerebrospinal fluid}

\section{Amanda Fitzpatrick, Lynda O'Leary, David Robertson, Isaac Garcia-Murillas, Andrew Tutt, Clare Isacke}

\section{Breast Cancer Now Research Centre, Institute of Cancer Research}

Introduction Breast cancer leptomeningeal metastasis (BCLM) is particularly devastating, with a median survival of just 3-4 months. Timely diagnosis is often challenged by low sensitivity of cerebrospinal fluid (CSF) cytology. Further, translational progress is hampered by the lack of in vivo BCLM models.

Methods CSF $(\leq 10 \mathrm{~mL})$ and plasma were collected from patients with confirmed BCLM. Following protocol optimisation, cell-free DNA (cfDNA) was extracted using Qiagen Circulating Nucleic Acid Kit, quantified by Qubit fluorometer and fragment length assessed by the Agilent Bioanalyzer. Primary human breast cancer cells were injected intravenously into NSG mice. Mouse skull caps were formalin-fixed before dissecting out the meninges, which were stained for human CK19 and lamin A/C, and whole-mounted for confocal immunofluorescent imaging. 
Major findings cfDNA was extracted from CSF and plasma in 5 confirmed BCLM cases (4 luminal and 1 HER2-enriched). Median cfDNA concentration was $9.3 \mathrm{ng} / \mathrm{mL}$ CSF (range 0.4-128.8 ng/mL). The dominant fragment length was $149-152 \mathrm{bp}$, in keeping with circulating tumour cfDNA size. Confocal microscopy revealed CK19 and lamin A/C-positive human tumour cells in the meninges, located close to the dural sinuses, and individual tumour cells tracking along vessel-like structures. Corresponding coronal brain sections revealed tumour growth on the perimeter but not within the brain parenchyma, in keeping with the pattern of leptomeningeal metastatic spread.

Significance of the research With the aim of developing a more sensitive diagnostic biomarker for BCLM, CSF cfDNA will be probed for known tumour mutations using digital PCR. To identify molecular drivers of BCLM, CSF cfDNA will undergo exome sequencing and comparison to plasma and primary tumour DNA. The in vivo model will be utilised for functional evaluation of identified molecular alterations, and for BCLM preclinical testing.

\section{Developing in vivo models to interrogate the mechanisms of breast cancer spontaneous brain metastasis}

\author{
Magdalena Kijewska, Marjan Iravani, Antoinette van \\ Weverwijk, Kuba Mieczkowski, Clare Isacke
}

The Institute of Cancer Research, Breast Cancer Now Research Centre

Introduction Brain metastases develop in 15-30 \% of metastatic breast cancer patients, and this number is rising due to advances in image-based detection and novel therapies that increase the survival of patients with secondary breast cancer. The mechanisms driving site-specific brain metastasis are mostly unknown. The major obstacle is the lack of appropriate animal models, and very low brain metastatic burden in existing models, which makes it difficult to study this disease.

Aims The aims of this project are to i) establish a spontaneous brain metastasis model in immunocompetent mice, ii) identify drivers of brain metastasis and iii) develop a method to quantify micro-metastases.

Procedures and Results We have developed a protocol where, using the 4T1 mouse mammary carcinoma cell line, we observe a frequency of spontaneous brain metastasis, which is representative of that seen in clinic. We have isolated 4T1 subclones derived from the brains, lungs and the primary tumours and performed gene expression profiling. Strikingly, the lung clones showed a near-identical profile to the tumour clones; however, the brain clones were clearly distinct from the lung and tumour clones. We have chosen the genes of interest to validate as potential drivers of brain metastasis and confirmed their differential expression in the independent brain clones from the 4T1 model and, in an alternative MDA-MB-231 spontaneous metastasis model, in $50 \%$ of brain subclones. To quantify the micrometastasis burden, we use flow cytometry and quantification beads that enable the absolute quantification of tumour cells in the brains. Conclusions We will use the established in vivo models and the tumour cell quantification, together with the molecular profiling of the metastatic subclones, to elucidate the mechanisms enabling breast cancer cells to spontaneously metastasis to the brain.

\section{The role of Kindlin-1 in the initiation and progression of breast cancer}

Val Brunton, Sana Sarvi, Morwenna Muir, Helen Creedon, Adam Byron, Hitesh Patel
University of Edinburgh

Kindlin-1 is a FERM domain-containing protein that is predominantly localised at focal adhesion sites where it interacts with the $\beta$-subunit of integrins and regulates their activity. It was identified as a gene whose loss or mutation is linked to Kindler syndrome, an autosomal recessive disease that leads to skin abnormalities including blistering and atrophy. However, overexpression of Kindlin-1 mRNA has been reported in colon and lung cancer and more recently Kindlin-1 mRNA expression was associated with metastasis-free survival in both breast and lung adenocarcinoma, although the mechanisms are not fully understood. To evaluate the role of Kindlin-1 in the initiation and progression of breast cancer, we used the MMTV-PyMT mouse model in which Kindlin-1 was specifically excised in the mouse mammary epithelium. Kindlin-1 deletion significantly delayed tumour onset and reduced lung metastasis, while primary tumour growth was unaltered. To address whether the reduced metastatic potential of Kindlin-1-depleted tumours cells was associated with defects in integrin activation, Kindlin-1 was deleted in the PyMT Met-1 cell line into which either wild-type Kindlin1 or a Kindlin-1 mutant (W612A) that is unable to activate integrins was re-introduced. Loss of Kindlin-1 reduced early lung colonization $30 \mathrm{~min}$ following intravenous injection of the cells. At this time, tumour cells have arrested in the lung vasculature and attached to the endothelial cells prior to extravasation. Re-expression of wild-type Kindlin-1, but not the W612A mutant, was able to rescue the lung colonization defect, demonstrating that the ability of Kindlin-1 to regulate lung colonization is integrin dependent. Kindlin-1 deletion reduced adhesion to both endothelial cells and the endothelial cell adhesion molecule VCAM in an $\alpha 4$ integrin-dependent manner suggesting that the decreased lung colonization in Kindlin-1-depleted cells is due to an early defect in tumour cell attachment to the endothelial cells within the lung.

\section{Clinical research benefits from patient involvement- the evidence}

Adrienne Morgan, Maggie Wilcox, Mairead MacKenzie, Lesley Turner, Jacqui Gath, and Pat Fairbrother

Independent Cancer Patients' Voice

Independent Cancer Patients' Voice is a patient advocate group led by patients for patients. We bring the views and experience of cancer patients, their families and carers, to the cancer research community. We believe that clinical research benefits from patients being partners with clinical researchers-rather than passive recipients of healthcare.

"The involvement of lay people in collections of tissue samples for research has been critical in allowing professionals to feel confident about what can reasonably asked of patients. Without this input, it is highly unlikely that our trials of presurgical treatments could have been successful."

Mitch Dowsett, Royal Marsden Hospital

"ICPV participation speeds up the process of turning a trial idea into a workable design, brings a welcome new perspective to the whole procedure and ensures the correct questions are asked."

Adele Francis University Hospital, Birmingham

"ICPV have been invaluable to our translational research program e.g. TRACERx, trial concept development, protocol writing and regulatory submission. Their network and attention to detail is unparalleled and helps us adapt to the needs of patients, rapidly accelerating approval processes and hastening trial recruitment."

Charles Swanton UCLH 
"ICPV has been vital in improving the POSNOC trial design, ensuring outcomes important to patients are measured."

Amit Goyal Royal Derby Hospital

"ICPV has influenced the design, information, consent, trial management and processes for PERSEPHONE. Also for OPTIMA and MAMMO-50 where ICPV organised PPI focus groups to determine the experiences and views of the trial design, information and trial question. Engagement of patients early in the trial design has helped with fine-tuning these important questions and has allowed the trial team to incorporate patients' view throughout the trial process."

Janet Dunn Warwick Trials Unit

"ICPV have been involved in the conceptual and practical development of the PEACE study from the outset. Their experience and input has been invaluable."

"Mariam Jamal-Hanjani, UCL"

\section{Comparing clinician and patient perspectives in the management of hot flushes in the UK breast cancer patients}

\author{
Adrienne Morgan ${ }^{1}$, Mei-Lin Ah-See ${ }^{2}$, Myra Hunter ${ }^{3}$, Jo Armes 3 , \\ Jacqueline Filshie $^{4}$ \\ ${ }^{1}$ NCRI CSG Working Party on Symptom Management; ${ }^{2}$ Mount
} Vernon Cancer Centre; ${ }^{3}$ Kings College London; ${ }^{4}$ The Royal Marsden

Women who have been treated for breast cancer identify vasomotor symptoms, such as hot flushes and night sweats (HFNS), as a serious problem. An estimated 550000 people live in the UK today with a diagnosis of breast cancer and up to $70 \%$ experience HFNS. Oestrogen replacement remains the most effective treatment for hot flushes. However, this is contraindicated in women with oestrogen-dependent breast cancer.

Fewer than $50 \%$ of women with ER+ breast cancer complete the recommended 5 years of endocrine treatment-tamoxifen and AIs. This lack of adherence, possibly due to unacceptable side effects, leads to a $20 \%$ excess breast cancer mortality.

Patient members of the NCRI Breast CSG identified that there is very little research into the management of symptoms after breast cancer treatment. In response, we established a Working Party on Symptom Management. We all have an interest in the management of HFNS, and members include patients; clinical and academic partners representing oncology, psychology, gynaecology, and complementary therapies; and the voluntary sector.

We gauged current clinical practice of the management of HFNS by surveying breast cancer patients, GPs and health professionals and we will present these data. There was a considerable mismatch between the three groups: for example, $40 \%$ of patients reported that no HPs or GPs had asked them about HFNS. Despite over $90 \%$ of GPs and HCPs reporting that they prescribed drugs to alleviate HFNS, only $26 \%$ of the patients had been offered drugs and fewer than $2 \%$ said they helped. $31 \%$ of the patients said that the HFNS were severe enough for them to consider stopping endocrine therapy. If women are to be helped to adhere to their life-saving treatment, new approaches need to be developed to ameliorate HFNS.

\section{What lies beneath the epigenetic signatures associated with breast cancer and how do we find out?}

\author{
Adele Murrell $^{1}$, Lovorka Stojic ${ }^{2}$ and Malwina Niemczyk ${ }^{2}$ \\ ${ }^{1}$ University of Bath; ${ }^{2}$ CRUK Cambridge Institute, University \\ of Cambridge
}

Epigenetic features such as DNA methylation status, histone modifications and chromatin states have been extensively profiled in the genome. Combinatorial epigenetic marks (epigenetic signatures) are emerging as breast cancer biomarkers and several studies are examining their diagnostic and prognostic value. However, very little is still understood about the mechanisms that underlie aberrant epigenetic programming in cancer cells. A specific example is long-range epigenetic silencing (LRES) where several contiguous genes are silenced simultaneously. We and others have shown that changes in chromatin conformation affects DNA methylation, histone modifications and the expression of genes within looping domains. New data from our studies in which genomic imprinting is used as a model system show that the expression of long noncoding RNA (lncRNA) can disrupt chromatin conformation and affect LRES through transcriptional interference. Since most of the genome actually consists of noncoding RNA, our results suggest that transcriptional interference and chromatin topology are interdependent. In addition to having cis functions as a result of their transcription, lncRNA can also have trans functions away from their site of transcription. Such trans functions are diverse but evidence is accumulating for lncRNAs having a role in recruiting chromatin remodelling proteins to specific regions of the genome. In a recent study in breast cancer cells, we were able to uncouple the cis and trans functions of a lncRNA and showed that inhibition of a lncRNA increases genes involved in the c-Met signalling pathway leading to increased cell migration and cytoskeletal changes. This example of a lncRNA and its cis and trans functions is a vignette of epigenetic remodelling affecting the invasiveness and metastatic potential of breast cancer cells.

\section{Modulation of EP300 alters paclitaxel resistance and is accompanied by regulation of cancer stem cell markers in breast cancer}

Roman Jugov, Muhammad Asaduzzaman, Stephanie Constantinou, Haoxiang Min, John Gallon, Meng-Lay, Charles Coombes, Eric Lam and Ernesto Yagüe

Imperial College London

There is an urgent need to understand how cancer cells spread and to identify markers of diagnostic and prognostic significance that can help in the development of novel therapeutics. We have recently described a novel pathway regulating drug resistance in breast cancer. The three miRs in the miR-106b 25 cluster (miR-106b, miR-93 and miR-25) target the $3^{\prime}$-UTR of EP300, a transcriptional activator of E-cadherin, down-regulating its expression and thus activating an EMT program accompanied by acquisition of cancer stem cell (CSC) properties and drug resistance.

Here, we have used a variety of breast cancer cell lines in which EP300 expression has been stably modulated (either down-regulated or overexpressed), as well as paclitaxel-resistant derivatives. We find that the absence of EP300 is directly linked to paclitaxel resistance and this is accompanied by an enrichment in breast cancer stem cell markers (i.e. enrichment of CD44high/CD24low sub-population and ABCG2). Importantly, cells in which EP300 has been either downregulated or knocked out show an up-regulation of antiapoptotic BCL2. Interestingly, ectopic expression of EP300 in breast cancer cell lines with mesenchymal characteristics (MDA-MB-231, CAL51) rescues the phenotype, with depletion of the CD44high/CD24low subpopulation and resensitization to paclitaxel. Transcriptome profiling of MCF-7 cells with EP300 down-regulation confirms that EP300 has an important role in regulating a plethora of molecules involved in drug resistance, motility, invasion and differentiation. 
Metaplastic breast carcinomas constitute a distinct aggressive form of invasive breast cancer characterized by lack of E-cadherin expression and elongated "fibroblast-like" cells. It is not a common type of breast cancer, but is highly aggressive and has a poor clinical outcome. We have analyzed by immunohistochemistry a small sample of metaplastic breast cancers and found EP300 highly downregulated, indicating that EP300 may play a role in the regulation of aggressive characteristics of this form of breast cancer.

\section{Epigenetic plasticity identifies seed breast cancer cells driving drug resistance and metastatic progression}

Sung Pil Hong, Ylenia Lombardo, Giacomo Corleone, Charles R. Coombes, and Luca Magnani

Department of Surgery and Cancer, Imperial College London, London, W120NN, UK

The Cancer Stem Cells (CSCs) hypothesis implies the existence of progenitor breast cancer cells with de novo resistance toward antioestrogen receptor (ER) therapies. Here we demonstrate that putative CSCs do respond to acute endocrine therapy but are uniquely predisposed to drive drug resistance and metastatic progression, using a noble endocrine therapy-resistant breast cancer cell model. After reprogramming, longterm oestrogen-deprived (LTED) cells, mimicking aromatase inhibitor (AI) resistance, acquire cell plasticity to recapitulate the whole population, while only CSC subpopulation of naïve breast cancer cells, MCF7 and T47D, show the plasticity. Interestingly, Tamoxifen- or Fulvestrantresistant cells do not acquire the plasticity. Upon acute oestrogen deprivation, CSCs become quiescent and require additional reprogramming to resume proliferation, while differentiated cells are efficiently cleared by oestrogen deprivation. Potential CSC subpopulations can be identified in patients with ER $\beta$-positive breast cancers where they are promptly selected by short-term neoadjuvant AI therapy and further expand in metastatic relapse following adjuvant treatment. Using ATAC-seq, we demonstrate that CSCs are epigenetically distinct and have more accessible regulatory regions than non-CSCs. The unidirectional differentiation is achieved by loss of regulatory regions, and this chromatin compaction involves enhancers and promoters of Notch target genes. These regulatory regions are maintained to be accessible in both subpopulations of LTED cells. Using low concentration of anti-Notch drugs, we could reduce the accessibility in the regulatory regions of Notch target genes, and force CSC differentiation into a targetable nonCSC subpopulation. Our data suggest that epigenetic plasticity contributes to drug resistance and might represent a novel therapeutic target.

\section{Cadherin-5 is a biomarker for metastatic breast cancer with optimum efficacy in oestrogen receptor-positive breast cancers with vascular invasion}

\section{Miriam Dwek, Ruth Swann, Claire Robertson, Simon Fry \\ Department of Biomedical Sciences, University of Westminster}

The identification of biomarkers for breast cancer prognostication and prediction is an important aim. We have been undertaking studies to identify proteins exhibiting alterations in glycosylation in breast cancer. A glycoproteomic study from our laboratory identified cadherin-5 (CDH5) as a serum marker of metastatic breast cancer. We validated the utility of $\mathrm{CDH} 5$ as a biomarker for breast cancer progression using a further series of serum samples collected from patients enrolled in the DietCompLyf study.
A nested case-control study design was used with serum samples from breast cancer patients who had developed a distant metastatic recurrence within five years post-diagnosis $(\mathrm{n}=52)$ and those who had remained recurrence free $(n=60)$. An ELISA was used to quantify the serum CDH5 levels and protein glycosylation was assessed by Helix pomatia agglutinin (HPA) lectin binding. Clinicopathological, treatment and lifestyle factors associated with metastasis and elevated biomarker levels were identified.

The CDH5 level $(\mathrm{P}=0.028)$ and ratio of CDH5:HPA binding $(\mathrm{P}=0.007)$ were elevated in breast cancer patients with metastatic disease. Multivariate analysis identified that the CDH5:HPA ratio and the formation of distant metastases were driven by patients with oestrogen receptor-positive (ER+) cancer with vascular invasion (VI+).

In conclusion, $\mathrm{CDH} 5$ levels and glycosylation distinguish patients with metastatic breast cancer from those that remain metastasis free. The test reached optimal sensitivity and specificity in ER-positive cancers with vascular invasion.

\section{Levels of Serpin B1 protein in white blood cells of breast cancer patients increase in metastatic breast cancer}

Lyudmila Pavlova ${ }^{1}$, Dawn Farrar ${ }^{1}$, Jay Mani ${ }^{1}$, Gergana Metodieva ${ }^{1}$, Metodi Metodiev', ${ }^{1}$, Mhil Murray ${ }^{2}$, Bruce Sizer ${ }^{2}$, Elena Klenova $^{1}$

${ }^{1}$ School of Biological Sciences of Essex, School of Biological Sciences, Wivenhoe Park, Colchester, Essex CO

The identification of novel biomarkers to predict the aggressiveness of breast cancer and supplement the current prognostic and treatment tools is an important clinical need. Interaction between the host's immune system and tumours has been known to be reflected in detectable changes in profiles of RNA and proteins in blood cells. Molecules differentially expressed in these cells may therefore have properties of cancer biomarkers. The aim of this investigation was to identify, validate and assess the biomarker potential of proteins differentially expressed in white blood cells (WBCs) of patients with primary and advanced breast cancer. Using integrated approaches (2D gel electrophoresis, high-throughput mass spectrometry and publicly available gene expression data), protein and gene expression profiles of WBCs were compared and a panel of 15 proteins with distinct expression patterns in WBCs of cancer patients was selected for further validation by RT-qPCR and Western blot assay. Our results accumulated so far demonstrate that one of the candidates, Serpin B1 (neutrophil elastase inhibitor), present in neutrophil granulocytes, has very promising biomarker potential. Thus, levels of Serpin B1 protein in WBC of breast cancer patients increase as the disease advances. Furthermore, gradual decrease of Serpin B1 levels in response to chemotherapy and endocrine treatment is associated with favourable prognosis. These data suggest the utility of Serpin B1 as a biomarker of poor prognosis and treatment efficacy. The biomarker properties of two other proteins identified in WBCs (Copine 3 and Lipocalin 2) are currently being investigated.

\section{Targeting PP2A to overcome resistance to HER2- targeted therapies in breast cancer}

Neil Conlon, Martina McDermott, Brigid Browne, John Crown and Norma O'Donovan

National Institute for Cellular Biotechnology, Dublin City University, Glasnevin, Dublin 
Aims HER2-targeted therapies, such as trastuzumab and lapatinib, have significantly improved the outcome for patients with HER2positive breast cancer. However, HER2-positive metastatic breast cancer frequently does not respond to these therapies or progresses after initial response. We have previously shown increased protein phosphatase 2A (PP2A) activity in lapatinib-resistant breast cancer and these cells are sensitive to PP2A inhibition. This study examined the PP2A inhibitor LB-100, which has recently been tested in a phase I clinical trial, in lapatinib-resistant breast cancer.

Procedures Proliferation assays, using acid phosphatase assay, were performed to assess sensitivity to HER2-targeted agents and LB-100. Immunoblotting was performed for p-eEF2, eEF2, PPP2CA, p-PPP2CA and PARP on lapatinib-resistant cells (SKBR3-L and HCC1954-L) and their parental cell lines. Cell cycle analysis was carried out using propidium iodide on a Guava EasyCyte. The effect of PP2A inhibition on the development of lapatinib resistance was examined by continuous exposure to lapatinib, LB-100 or combination until lapatinib-treated cell regrowth. Crystal violet staining and quantification were used to assess cell growth.

Major findings Both lapatinib-resistant cell lines showed decreased sensitivity to HER2-targeted agents, including neratinib and afatinib. Both cell lines had decreased p-eEF2 and p-PPP2CA levels. These cells were significantly more sensitive to LB-100 than their parental cells and LB-100 had a synergistic effect in combination with lapatinib in both cell lines. LB-100, as a single agent and in combination with lapatinib, caused an increase in cells in SubG1 and increased PARP cleavage, indicating apoptosis.

Significance of research This study shows that the therapeutic PP2A inhibitor LB-100 may have clinical efficacy in the treatment of refractory HER2-positive breast cancer.

Conclusions PP2A inhibition, with LB-100, causes apoptosis in lapatinibresistant cells and enhances response to lapatinib. Therefore, PP2A represents a novel target for lapatinib-resistant HER2-positive breast cancer.

\section{Defining novel stromal and tumour characteristics associated with response to neoadjuvant chemotherapy in breast cancer}

Celine Pourreyron, Shelley Waugh, Patsy Whelehan, Philip Coates, Lee Jordan, Colin Purdie, Sarah Vinnicombe, Alastair Thompson, Andrew Evans, Frances Fuller-Pace

\section{University of Dundee}

Introduction Neoadjuvant chemotherapy (NACT) is commonly used in breast cancer treatment to decrease tumour and lymph node burden and the extent of surgery. However, response is variable and prediction poor. Our programme aims to identify peritumoural and tumour imaging signals and stromal gene signatures which predict response to NACT and could be used to monitor and adapt NACT.

Methods 39 patients have undergone ultrasound shear-wave elastography and 3T MRI (including textural analysis [TA], reflecting tumour heterogeneity, and functional tumour volume [FTV] estimations) before, after 3 and after 6 cycles of NACT. Illumina gene expression microarray was performed using primary stromal cells isolated from peritumoural and tumour pre treatment core biopsies from 8 pathological responders and 9 non-responders to NACT. Differentially expressed genes (fold change $\geq 1.2, p$ value $<0.05$ ) were identified with $\mathrm{MeV}$ software. Gene expression profiles and changes in imaging parameters are compared with percentage cellularity reduction and the residual cancer burden (RCB) on final resection pathology.

Results Initial results show that percent reduction in FTV at interim MRI was associated with pCR (78\% sensitivity and $89 \%$ specificity). Changes in TA are also associated with a greater reduction in heterogeneity seen in good responders (pCR, RCB-1), whereas conventional RECIST criteria performed poorly. Reduction in peritumoural stromal stiffness showed $62 \%$ sensitivity and $95 \%$ specificity for pCR. Expression profiles revealed more dysregulated genes between the peritumoural stromal cells of responders versus non-responders than in the corresponding tumour cells.

Conclusions Novel ultrasound and MRI imaging parameters are superior to standard imaging methods in assessing interim response to NACT. Gene expression discrimination between good and poor responders to NACT is more pronounced in peritumoural stroma than in the cancers. These pilot data provide opportunities for predictive testing within future clinical trials.

\section{Engineering Mesenchymal Stem Cells (MSCs) for exosome mediated delivery of microRNAs} Killian O'Brien, S. Khan, D.P. Joyce, P. Lalor,
P. Dochery, M.J. Kerin, R.M. Dwyer

Discipline of Surgery, NUI Galway

Exosomes are small membrane-derived vesicles that are secreted by most cells and are readily taken up by recipient cells. Exosomes selectively package genetic material such as microRNAs, important regulators of genes involved in breast cancer progression. Mesenchymal Stem Cells (MSCs) are multipotent stromal cells known to migrate and engraft into the tumour microenvironment where they play a key role in intercellular communication. MSCs offer potential as vehicles for targeted delivery of therapies. Engineering MSC-derived exosomes secreted within the tumour microenvironment holds exciting potential. The aim of this study was to investigate exosome-mediated cross-talk between MSCs and breast cancer cells and to further engineer the microRNA content of MSC-derived exosomes.

Methods MSCs were transduced with a lentivirus containing a tumour suppressor microRNA or a control sequence, both were tagged with a red fluorescent protein (RFP). Exosomes were isolated from cell-conditioned media by differential centrifugation, microfiltration and ultracentrifugation. Exosomes were characterised by transmission electron microscopy(TEM). The exosome-encapsulated microRNA was subsequently extracted and analysed by RQ-PCR. The impact of MSC-derived exosomes on breast cancer cell proliferation was determined.

Results Exosomes were successfully isolated from the MSCs. Expected size and morphology were confirmed by TEM. Exosome transfer onto recipient breast cancer cells was confirmed by confocal microscopy. Exosomal cross-talk between wild-type MSCs and breast cancer cells did not significantly impact proliferation. MSCs were successfully engineered to express 250-fold higher levels of the target microRNA. Exosomes from engineered MSCs that expressed the RFP tag of the parent cell were efficiently taken up by breast cancer cells, and also demonstrated a fivefold increase in the expression of the target microRNA.

Conclusion Wild-type MSC-derived exosomes did not impact breast cancer cell proliferation. It is also possible to engineer MSC-derived exosomes which has exciting potential as a clinically relevant means of therapeutic delivery.

\section{HER2 expression levels of breast cancer cell lines grown in $2 \mathrm{D}$ and $3 \mathrm{D}$ cell culture and sensitivity to chemotherapeutic drugs}

Tayebeh Azimi, Miriam Dwek, Anatoliy Markiv 
University of Westminster

Recently, with the advent of different methods of 3-dimensional (3D) cell culture, scientists can now address some of the limitations of conventional 2D methods. 3D cell culture systems attempt to mimic the in vivo tumour microenvironment more accurately particularly with respect to the gene expression profile and response to treatment. HER2 amplification occurs in approximately $20 \%$ of invasive breast cancers and is associated with poor prognosis and reduced overall survival.

In this study, four breast cancer cell lines which have different ER/ PR and HER2 gene expression profiles have been grown under 2D and 3D cell culture conditions. The HER2 level has been analysed in both conditions using mRNA and protein and by fluorescent cell imaging. Cells were treated with the anti-HER2 drug trastuzumab and with the chemotherapeutic agent doxorubicin and assessed using Alamar blue as a measure of cell viability. The results show that gene regulation and drug efficacy are affected according to the microenvironment in which the cancer cells are maintained.

\section{Molecular changes in premenopausal ER+ breast cancer after ovariectomy}

Ben Haynes, O. Ginsburg, A. Gao, E. Folkerd, M. Afentakis, Han Thi Pham, Le Hong Quang, Ta Van To, Nguyen Van Dinh and M. Dowsett

The Ralph Lauren Centre for Breast Cancer Research, The Royal Marsden Hospital and Institute of Cancer Research, London SW3 6JJ

Aim To characterize the molecular effects of neoadjuvant ovariectomy (OvX) in ER+ breast cancers.

Background For premenopausal women, OvX is an evidence-based cost-effective option with survival rates comparable to first-generation chemotherapy and is standard treatment in many countries. There are virtually no data describing the biological effects of OvX in premenopausal breast cancer.

Patients and Methods A non-randomized phase II trial of neoadjuvant OvX was conducted in 56 premenopausal women with ER+ breast cancer in Vietnam. Serum and tumour samples were taken at diagnosis, oophorectomy $(\mathrm{t}=2$ weeks $)$ and mastectomy $(\mathrm{t}=4$ weeks). Serum hormone concentrations were measured by RIA. Ki67, ER, PgR and HER2 were measured by IHC. RNA was subject to whole-genome expression profiling (32 pairs).

Results Estradiol concentrations decreased from $421 \pm 305$ to $24.1 \pm 24.5 \mathrm{pmol} / \mathrm{l}$ (mean $\pm \mathrm{SD}) \quad 24 \mathrm{~h}$ after OvX and to $8.8 \pm 7.3 \mathrm{pmol} / \mathrm{l}$ by mastectomy. Ki67 decreased in 33/36 (91.7 \%) of tumours: the changes did not differ according to HER2 status. After OvX the expression of 756 genes changed significantly $(p<0.005)$ with fold change $(\mathrm{FC})>1.25$. TFF1 was the most highly down-regulated gene (FC 0.20; $<$ 1e-07); other oestrogen-regulated (e.g. GREB1, PDZK1, PGR) and proliferation-associated genes (e.g. TOP2A, CDC20) also showed large decreases in expression (FC 0.41-0.69: $\mathrm{p}<1 \mathrm{e}-07$ ). Progesterone-regulated genes, e.g. FKBP5, ESRRA, were also downregulated. Changes in gene expression after OvX strongly correlated with those after aromatase inhibitor (AI) treatment in postmenopausal women $(r=0.87, p<1 e-04)$ [1]. After OvX, more than twice as many genes showed a FC $>1.25$ (721 vs. 331$)$ and the mean FC was significantly higher $(\mathrm{p}<1 \mathrm{e}-04)$ compared to AI.

Conclusion Molecular changes in ER+ breast cancer after OvX in premenopausal women are similar to those after AI in postmenopausal patients. However, OvX affects more genes and has a greater impact on them.

[1] Clinical Cancer Research 2013;19:2775
Use of CRISPR-Cas9-mediated knockin mutagenesis of ESR1 mutations in metastatic breast cancer allow functional analysis and evaluation of treatment options

Joel Fulton, Alison Harrod, Van Nguyen, Manikandan Periyasamy, Laura Ramos Garcia, Chun-Fui Lai, Alex de Giorgio, Gergana Metodieva

Imperial College London

As the major driver of breast cancer (BC) development and progression, $\mathrm{ER} \alpha$ is the predominant target for adjuvant therapies. Inhibition of $\mathrm{ER} \alpha$ activity with anti-estrogens or inhibition of estrogen biosynthesis (aromatase inhibitors, AI) reduces relapse and improves patient survival. However, many patients with ER $\alpha$-positive BC relapse on these therapies, resistant tumours mostly remaining $\mathrm{ER} \alpha$ positive. Efforts to identify changes in $\mathrm{ER} \alpha$ that functionally act in resistance show that mutations in the ER $\alpha$ gene are exceptionally rare in primary BC. By contrast, recent discoveries show that $\mathrm{ER} \alpha$ is frequently mutated in Endocrine-resistant metastatic breast cancer. These reports provide strong evidence for treatment-selective pressure leading to acquisition of $\mathrm{ER} \alpha$ mutations. To determine the function of these mutations, we used the CRISPR-Cas9 system to generate the common (L536R, Y537S, Y537 N, Y537C, D538G) mutations in the genetically encoded ER $\alpha$ gene in MCF7 cells. Evaluation of ER $\propto$ recruitment to DNA (ChIP-seq) and expression profiling (RNA-seq) demonstrate that the mutations result in estrogen-independent expression of many ER $\alpha$ target genes, accompanied by estrogen-independent growth and partial resistance to anti-estrogens. We will further also present evidence that inhibitors of key signalling pathways regulating ER $\alpha$ activity provide a means of restoring anti-estrogen responsiveness. Therefore, these mutant breast cancer cell lines provide an important resource for determining approaches for the treatment of breast cancer featuring ER $\alpha$ mutations.

\section{WSB-1 regulates the metastatic potential of hormone receptor-negative breast cancer}

Isabel Pires, Flore-Anne Poujade, Lisa Eigleman, Aaren Mannion, Ellie Beeby, Sage Pickwell, Andrew Theodosi, Christopher Cawthorne

University of Hull

Metastatic burden is responsible for the majority of cancer-associated deaths. Therefore, it is of utmost importance to clarify the mechanisms underlying tumour cell spread. This will allow the development of better cancer prognostic tools and therapeutic approaches. The tumour microenvironment is a major driver of metastatic disease. Particularly, hypoxia is associated with therapy resistance and increased tumourigenic and metastatic potential. Although some of the factors important for regulation of hypoxia-associated metastasis have been identified, many aspects of this phenomenon still remain unclear.

The aim of this study was to investigate the role of the poorly characterised WSB-1 (WD40-repeat and SOCS Box containing-1) E3 ligase in breast cancer biology, particularly in the context of tumour microenvironment conditions, such as hypoxia.

We have identified WSB-1 as a hypoxia-inducible factor in a wide variety of breast cancer cell lines. In silico analyses indicated that high WSB-1 expression was associated with decreased distant metastasisfree survival and relapse-free survival in a cohort of ER- and PR-negative breast cancer patients. Knockdown of WSB-1 expression led to decreased in vitro cellular migration and invasion, as well as angiogenic 
potential, but only for ER- and PR-negative cell line models. This was associated to altered MMP expression and activity, as well as decreased expression and secretion of VEGF. Importantly, knockdown of WSB-1 was also associated with decreased metastatic burden for in vivo experimental models of metastasis.

The present study indicates that the hypoxia-inducible factor WSB1 has a role in regulating metastatic spread in breast cancer, specifically in the context of hormone receptor biology. Further clarification of the signalling pathways involved in mediating WSB-1 function will clarify novel aspects of aggressive metastatic disease in breast cancer.

\section{Pro-inflammatory cytokines promote adhesion of breast tumour cells to brain endothelium}

\section{Ester Pascual-Baixauli, C. Cerutti ${ }^{1}$, E. Garattini ${ }^{2}$, I.A.Romero ${ }^{1}$,} F. $\mathrm{Crea}^{1}$

Department of Life Health and Chemical Sciences, the Open University, Milton Keynes MK7 6AA UK;

${ }^{1}$ The Open University, Milton Keynes;

${ }^{2}$ Mario Negri Institute, Milan, Italy

Metastatic spreading is the main cause of death in breast cancer patients. Brain metastases are resistant to most available treatments and associated with particularly poor prognosis. The development of metastases is a multi-step process. While most of these steps have been extensively studied, the adhesion of cancer cells to the vascular endothelium is not well characterised. Pro-inflammatory cytokines (IFN $\gamma / \mathrm{TNF} \alpha$ ) promote breast cancer dissemination to the brain. However, the mechanisms and clinical significance of this phenomenon have not been elucidated.

We developed a micro-fluidic system that enables us to measure the adhesion of breast cancer cells to brain endothelial cells (hCMEC/ D3), under shear stress conditions. We exposed hCMEC/D3 cells to IFN $\gamma$ and $\mathrm{TNF} \alpha(1 \mathrm{ng} / \mathrm{ml})$ or vehicle and measured the relative adhesion of breast cancer cells. We found that cytokines significantly enhanced the adhesion of MDA-MB-157 cancer cells to hCMEC/D3 cells $(>50$-fold change vs. control, $\mathrm{P}<0.05$ ). This result has been replicated in $\mathrm{HCC} 1954$ and $\mathrm{HCC} 114 \mathrm{vvc} 3$ cells. Our proteomics analysis revealed that IFN $\gamma$ and TNF $\alpha$ significantly up-regulated the expression of VCAM1 and LGALS9 cell adhesion molecules in hCMEC/D3 cells $(3.219 \pm 0.372,2.338 \pm 0.595$ fold change vs. control, respectively).

In order to identify the molecular mechanisms of cancer/endothelium interaction, we employed a pathway analysis software and identified putative ligands of VCAM1 and LGALS9, then examined the expression of these ligands in a database that includes 1609 breast cancer clinical samples. We found that the ITGA9/ITGB1 dimer (VCAM1 ligand) and the P4HB protein (LGALS9 ligand), are highly expressed in breast cancer cells. More importantly, ITGA9/ITGB1 and $\mathrm{P} 4 \mathrm{HB}$ up-regulation predicted shorter metastasis-free survival in patients (log-rank p values $=0.0011$ and 0.0066 ; hazard ratios: 1.43 and 1.34 , respectively).

These results indicate that pro-inflammatory cytokines could promote breast cancer metastatic tropism via up-regulation of specific cell adhesion proteins.
Impact of tyrosine kinase expression levels in human breast cancer cells on their response to treatment with combinations of small-molecule tyrosine kinase inhibitors

\author{
Aryan Stanley, Helmout Modjtajhedi
}

Kingston University

Despite recent advances in diagnosis and treatment, breast cancer remains one of the leading causes of mortality worldwide, highlighting the need for development of more effective therapeutic interventions and the identification of more reliable predictive markers of response to therapy. Here, we investigated the sensitivity of a panel of breast cancer cell lines to treatment with various tyrosine kinase inhibitors (TKIs), including reversible and irreversible ErbB-family inhibitors, and inhibitors of Src/Abl (dasatinib) and c-Met/ALK (crizotinib), as single agents and in combination, and whether there was any association between expression level of target antigens and response to treatment. The effects of these agents on cell downstream signalling and tumour migration were also investigated using Western blot and cell migration assay. Of the ErbB-family inhibitors, the irreversible pan-ErbB inhibitors were generally more effective at inhibiting cell growth than their reversible counterparts. We found the combinations of ErbB-family inhibitors with dasatinib were synergistic in the HER-2- and EGFRoverexpressing BT474 and MDA-MB-468 cells, respectively, both of which also had high levels of pSrc. Interestingly, the same combinations were antagonistic in SKBr3 cells which also had HER-2 overexpression but lower levels of pSrc. When the ErbB-family inhibitors were combined with crizotinib, their effects were mainly synergistic in MCF7 and MDA-MB-468, but mainly antagonistic in the HER-2-overexpressing SKBr3 and BT474 cells. Of all the TKIs examined, we found canertinib, dastinib, sapitinib and afatinib to be the most effective for reducing the migration of highly migratory MDA-MB-231 cells. Finally, with the exception of HER-2 and canertinib, we did not find a significant association between the expression level of other target antigens and response to these treatments. Our results suggest that while these inhibitors may have improved efficacy in combination, further investigations are warranted for the prediction of response to these therapeutic interventions in breast cancer.

\section{Targeting breast cancer stem cells through their P-Rex1/Rac1 signalling}

\section{Ahmet Ucar, David Novo, Keith Brennan, Rob Clarke, Charles Streuli}

University of Manchester

Breast cancer is the second most common cause of death from cancer in women in the UK. Although conventional and targeted therapies can prolong disease-free survival of patients, acquired drug resistance and tumour relapse are frequently observed in clinics. At the core of this clinical problem are breast cancer stem cells (Br-CSCs), a small fraction of tumour cells with stemness features and the capacity to re- 
initiate tumour formation. Br-CSCs are likely to be responsible for primary tumour formation, metastasis, acquired drug resistance and tumour recurrence. Using pharmacological inhibitors, we have determined that the activity of the small GTPase Rac1 is essential for the self-renewal and maintenance of Br-CSCs. Additionally, we have used the CRISPR-Double Nickase approach of genome engineering to address the roles of Rac1 signalling pathway in Br-CSCs. We have determined that loss of function of P-Rex1, an upstream regulator of Rac1 that is highly expressed in luminal breast tumours, results in premature differentiation and thereby depletion of Br-CSCs. We have also shown that the tumour-specific splice variant of Rac1, Rac1b, regulates $\mathrm{Br}$-CSC plasticity. Moreover, genetic deletion of Rac1b leads to a transition of Br-CSCs into a chemotherapy-sensitive state. Our study reveals that $\mathrm{P}-\mathrm{Rex} 1$ and Rac $1 \mathrm{~b}$ are two attractive molecular targets for the elimination of Br-CSCs.

\section{A lump and a bump: My experience of the breast cancer journey while pregnant and with a young baby}

\section{Cassandra Tait, Patient Advocate}

When diagnosed with breast cancer while 13 weeks pregnant, the lack of experience of pregnancy in cancer practitioners (and of cancer in antenatal practitioners), their reluctance to commit to treatment plans and the consistent changes of plans for baby and myself were apparent. My journey through treatment became one of isolation, fear and confusion as treatment plans changed and birth plans were not considered. The rarity of breast cancer during pregnancy became clear when my story began with "it's just a blocked milk duct", included a nurse telling me he felt uncomfortable giving me chemotherapy while pregnant, a 2-week stay in intensive care for my newborn baby, nightfeeds following chemotherapy cycles and is now coming to a hopeful conclusion with an ongoing battle between effective and meaningful parenting and debilitating fatigue.

My experiences highlighted a need for patients to be put in touch with others experiencing the same or similar to avoid isolation. In addition, there is a gap in the psychological services available to women in the specific position of fearing for the life of their unborn child, as well as their own. Moreover, the complexity of the side effects of treatment appears heightened when there is a need to care for a very young baby (and 4 years old).

\section{Endothelial HIF-a modulate cancer metastatic potential by affecting vascular permeability and pro-metastatic macrophage infiltration}

\section{Cristina Branco, Colin Evans, Renato Colaco, Helene Rundqvist, Randall Johnson}

\section{University of Cambridge}

Endothelial cells play a central role in the process of metastases, as the endothelium is the first physical barrier separating the primary tumor from the rest of the organism. To prevent metastatic events is a major concern in breast cancer treatments, as this is the phenomenon ultimately associated with mortality and morbidity. We have previously shown that deletion of endothelial Hypoxia-Inducible Factors (HIFs) strikingly affects metastatic success in an isoformspecific manner (Branco-Price et al., Cancer Cell, 2012). In this work, we show that acute hypoxia predisposes lungs for metastatic colonization. This correlates with increased HIF-1a, increased endothelial permeability, and increased levels of MCP1, which we believe is secreted, to a significant extent, by endothelial cells. Chronic hypoxia, however, does not seem to predispose lungs for tumor growth in any manner different from normoxic preconditioning, in spite of increased macrophage infiltration into lungs. Our observation suggests that a combination of increased permeability and specific prometastatic macrophage infiltration enhances metastatic potential, in a process that is mediated by endothelial HIF-1a, and we can reverse this phenomenon in mice lacking lung endothelial HIF-1a. In chronic conditions, where HIF-2a is preferentially activated and HIF-1a is less prevalent, mice develop secondary tumors to an identical extent to those kept in normoxia. We are further exploring the consequences of vascular occlusion and localized hypoxic insult, as seen during circulating tumor cell arrest in small capillaries, having observed that an acute and localized hypoxic insult following inflammation has a potentiating effect in metastatic proliferation, whereas a microocclusion that occurs at the same time or earlier than tumor cell injection has no effect. The mechanisms underlying this phenomenon appear to depend on both endothelial and inflammatory responses: a fine balance in endothelial activation, inflammatory components, and dynamic stabilization of HIF isoforms that significantly affect metastatic predisposition.

\section{ICEC0942, an orally bioavailable selective inhibitor of CDK7 for breast cancer}

\author{
Simak Ali ${ }^{1}$, Hetal Patel ${ }^{1}$, Manikandan Periyasamy ${ }^{1}$, Alexander \\ Bondke $^{2}$, Brian W. Slafer ${ }^{2}$, Silvia Ottaviani ${ }^{1}$, Alison Harrod ${ }^{1}$, \\ Laki Buluwela $^{1}$, Matthew J. Fuchter ${ }^{2}$, Anthony, G. M. Barrett ${ }^{2}$, \\ R. Charles Coombes ${ }^{1}$
}

${ }^{1}$ Department of Surgery \& Cancer; ${ }^{2}$ Department of Chemistry, Imperial College London, UK

Estrogen receptor- $\alpha$ (ER) is the transcriptional driver in the majority of breast cancers. ER activity is targeted with inhibitors of estrogen biosynthesis or with anti-estrogens, such as Tamoxifen. While these drugs have been extremely successful, many patients relapse, with few treatment options being available following emergence of resistance. Recent reports show that many cancer types are highly sensitive to transcription inhibition, suggesting that targeting the transcriptional machinery provides new approaches to cancer treatment. Cyclin-dependent kinase (CDK)7, which is required for RNA polymerase II-mediated transcription, also regulates the activities of several transcription factors, including ER. We have identified a new selective inhibitor of CDK7, ICEC0942. In vitro studies show that a wide range of cancer types are sensitive to CDK7 inhibition. In xenografts of both breast and colorectal cancers, the drug has substantial antitumour effect. Additionally, combination therapy with tamoxifen showed complete inhibition of ER-positive tumor xenografts. Our findings reveal that CDK7 inhibition provides a new approach for treatment of ER-positive breast cancer and identify ICEC0942 as a prototype drug with potential utility as a single agent or in combination with hormone therapies. 


\section{Targeting folate receptor alpha for breast cancer treatment}

\author{
Anthony Cheung, James Opzoomer, Kristina Ilieva, Rebecca \\ Marlow, Erika Francesch Domenech, Matthew Fittall, Patrycja \\ Gazinska, Natalie Woodman, Gyula Petranyi, Nirmesh Patel, \\ Hasan Mirza, Sarah Pinder, Cheryl Gillett, Anita Grigoriadis, \\ Frank Nestle, James Spicer, Andrew Tutt and Sophia \\ Karagiannis
}

\section{King's College London}

Promising targeted treatments and immunotherapy strategies in oncology and advancements in our understanding of molecular pathways that underpin cancer development have reignited interest in the tumour-associated antigen Folate Receptor alpha (FR $\alpha)$. FR $\alpha$ is a glycosylphosphatidylinositol (GPI)-anchored membrane protein. Its overexpression in solid tumours such as ovarian, breast and lung cancers and low and restricted distribution in normal tissues, alongside emerging insights into tumour-promoting functions, together render FR $\alpha$ an attractive therapeutic target.

We demonstrated expression of FR $\alpha$ in basal subtypes of breast cancer in the King's College London and METABRIC genomic datasets. In combination with RNA interference experiments, we showed that the inhibition of FR $\alpha$ reduced cellular proliferation, colony formation and ERK activation in FR $\alpha$-expressing breast cancer cell lines.

A monoclonal antibody targeting $\mathrm{FR} \alpha, \mathrm{MOv} 18 \mathrm{IgE}$, is already in a phase 1 clinical trial in ovarian cancer, and is being explored in our laboratory as a novel first-in-class IgE immunotherapy for the treatment of breast cancers. Using a multi-colour flow cytometry-based in vitro tumour cell killing assay, we demonstrated the ability of MOv18 to induce antibody-dependent cell-mediated cytotoxicity (ADCC) against FR $\alpha$-expressing breast cancer cell lines by human monocytes and healthy volunteer peripheral blood mononuclear cells. In addition, we evaluated expression of FR $\alpha$ in immuno-humanized xenograft models using immunohistochemistry and we found potential models for further in vivo efficacy studies.

In summary, our findings demonstrate that the tumour antigen FR $\alpha$ is expressed in basal breast cancers and its expression may support cancer cell growth. Functional studies with an anti-FR $\alpha$ monoclonal antibody warrant further characterisation of this antigen as a potential new therapy target in subsets of breast cancer, especially triple-negative breast cancers, for which effector cell infiltration is already known to be favourably prognostic.

\section{Breast Cancer Now Cell Culture Programme: a bespoke resource for the research community}

\section{J. Louise Jones, Jenny Gomm, Linda Haywood, Adrienne Morgan, Iain Goulding, Mike Allen, Claude Chelala, Emanuela Gadelata}

Barts Cancer Institute, Queen Mary University of London

The Breast Cancer Now Cell Culture Programme is part of the Breast Cancer Now Tissue Bank and is a unique initiative aimed at providing primary cells and 'live' tissues to the research community. The Programme utilises a broad range of tissues including normal breast tissue, prophylactic mastectomy tissue from high-risk individuals, including BRCA1 and BRCA2 mutation carriers, breast cancer tissue and matched material taken adjacent to $(<2 \mathrm{~cm})$ and distant from $(>5 \mathrm{~cm})$ the cancer, as well as tumour cells derived from lymph node metastases. Tissue is digested and different cell populations isolated, including from non-tumour tissues luminal epithelial, myoepithelial and fibroblast populations. From tumour-derived samples, fibroblasts and tumour cell-enriched populations are generated. In some cases, samples are digested only to organoid stage and can be provided as such, or single-cell unsorted preparations can be provided for subsequent sorting and characterization. Sample sets can be provided, including matched tumour-associated fibroblasts and surrounding non-tumour fibroblasts, matched tumour cell-enriched preparations and fibroblasts, and matched non-tumour-associated cells. In addition to cell preparations, explant culture material also is available: pieces of tumour or non-tumour material, $2 \times 2 \times 2 \mathrm{~mm}$, that can be cultured for varying periods (currently data from samples cultured between 5 and 25 days) under different conditions. All samples have linked comprehensive clinicopathological information and can also be linked to matched tissue samples from the Tissue Bank.

The Breast Cancer Now Cell Culture Programme also operates an $R \& D$ programme to enhance the utility of the samples provided. This includes culture of cells on different matrices and scaffolds, extensive biomarker characterization, and an immortalization schedule, to increase the availability and longevity of the samples. Selected samples are currently undergoing Whole-Genome Sequencing and these data will become available through the BCNTN Bioinformatics Portal.

\section{Breast cancer and return to work: a pilot}

\section{Julie Denning, N. Hunter}

Working Towards Wellbeing

Around 100,000 people of working age are diagnosed with cancer yearly. However, as treatments for cancer improve, increasing numbers of people become 'cancer survivors', wanting to return to normal functioning including work; however, they are 1:4 more likely to be unemployed than matched controls. Work has been shown to be good for health (Burton, Waddell and Kendal, 2008) and improves function and general wellbeing post diagnosis.

Research suggests that people surviving cancer want to return-towork (Amir et al., 2008, Macmillan, 2008). However, most rehabilitation services do not address return to work or the associated physical, emotional and practical issues affecting cancer survivors such as fatigue, pain, financial problems and reduced self-efficacy.

Providing support through multilevel case management is predicted to help people on their return-to-work journey as research by Macmillan has shown that such Vocational Rehabilitation (VR) services are beneficial. This research aims to build on their findings by providing a stepped care model within a population of income protection claimants to ascertain whether a VR service can have a positive impact on returnto-work outcomes and physical and psychological factors.

A longitudinal pilot study of 32 people was carried out to explore improvements in mood, fatigue, quality of life, self-efficacy, readiness to return to work and satisfaction.

$58 \%$ of the group returned to work. There was a $34 \%$ improvement in the Chalder Fatigue Scale, $48 \%$ improvement in the GAD7, $52 \%$ improvement in the PHQ9, $11 \%$ improvement in Selfefficacy Scale, and a $32 \%$ improvement quality of life.

The findings from this pilot study are positive and suggest that there is a need for VR services for people who have survived cancer to improve their quality of life including return-to-work plans. 


\section{Cytidine deamination activity of APOBEC3B regulates estrogen receptor function in breast cancer}

Manikandan Periyasamy, Van T. M. Nguyen, Hetal Patel, ChunFui Lai, Ekaterina Nevedomskaya, Alison Harrod, Laki Buluwela and Simak Ali

\section{Imperial College London}

We have discovered that APOBEC3B (A3B), recently linked to the mutational landscape in breast and other cancer types, is required for breast cancer cell growth. Using ChIP-seq and RNA-seq profiling, we have shown that $\mathrm{A} 3 \mathrm{~B}$ is recruited to the majority of ER-binding regions in ER+ breast cancer cells, to regulate ER target gene expression. Mechanistic studies established that A3B regulates gene expression causing $\mathrm{C}$-to- $\mathrm{U}$ deamination at ER-binding regions. We show that these C-to-U changes lead to the generation of DNA strand breaks through activation of base excision repair (BER) and to repair by non-homologous end-joining (NHEJ) pathways. These studies demonstrate that the transient cytidine deamination by A3B aids chromatin modification and remodeling at the regulatory regions of ER target genes, which promotes their expression. Furthermore, extending the genomic profiling to other breast cancer cell lines shows that A3B is an important transcriptional regulator in ER-positive and in ER-negative breast cancer. Furthermore, these findings also provide insights into potential mechanisms by which transcription factor-mediated recruitment of $\mathrm{A} 3 \mathrm{~B}$ to DNA may facilitate cancer mutations by A3B.

\section{Investigating the role of RUNX2 in the breast cancer microenvironment}

\author{
Nicholas Rooney ${ }^{1}$, Laura McDonald ${ }^{1}$, Joanna Morris ${ }^{2}$, Ewan \\ Cameron $^{2}$, Joanne Edwards ${ }^{2}$, Karen Blyth ${ }^{1}$ \\ ${ }^{1}$ CRUK Beatson Institute, Bearsden, Glasgow, G61 1BD; ${ }^{2}$ University \\ of Glasgow, Garscube Estate, Bearsden, Glasgow, G61 1QH
}

The stromal microenvironment associated with epithelial tumours is known to have a profound influence on the transformation, progression and dissemination of cancers. Currently, little is known about the transcription factors within stromal cells that are responsible for regulating these processes. RUNX2 is a highly conserved transcription factor that is absolutely required for normal mammalian bone development. RUNX2 has also been shown to be expressed in the normal mammary gland and has been implicated in breast cancers, where it is highly expressed in metastatic breast cancer cell lines. To date, very little is known about the role of RUNX2 within the mammary stromal microenvironment and what effect this has in the context of breast cancer. Here we confirm in an analysis of a human tissue microarray from 417 patients with primary breast cancer that RUNX2 is more highly expressed in the stroma of breast cancers compared to the epithelium and that this high expression correlates with poor prognosis in ER-negative tumours. In addition, we show that RUNX2 is clearly expressed in the stroma of tumours derived from the MMTV-PyMT (mouse mammary tumour virus-polyoma middle $\mathrm{T}$ antigen) GEM model of breast cancer. Immuno- histochemical analysis shows that RUNX2 expression correlates with fibroblast markers rather than immune cell markers. We then used FSP-Cre (fibroblast-specific protein-1) to specifically delete one copy of Runx 2 in the PyMT model (FSP-Cre+; PyMT+; Runx $2^{\mathrm{fl} /+}$ ). This revealed that heterozygous deletion of Runx2 in fibroblasts increases the average lifespan of the mice and improved overall survival. FSPCre + ; PyMT + ;Runx $2{ }^{\mathrm{fl} /+}$ mice also had increased tumour-free survival, and a delay in tumour progression to clinical endpoint compared to wild-type FSP-Cre+; PyMT+; Runx $2^{+/+}$mice. Together, this indicates that Runx 2 is highly present in the stroma of breast cancers and that reduction in its stromal expression can delay breast cancer progression.

\section{Circulating nucleic acids for monitoring metastatic breast cancer}

\section{Allison Hills}

Currently, there is a limited clinical ability to monitor breast cancer patients for signs of drug resistance at the molecular level. Development of minimally invasive blood-based assays, with a focus on Circulating Tumour Cells (CTCs) and circulating cell-free DNA (cfDNA), offers opportunities for real-time monitoring and treatment stratification that could significantly impact on patient outcome. CfDNA is potentially easier to obtain than CTCs and so it is important to determine which, if either, the more suitable candidate for clinical use is. The purpose of this study was to directly compare the mutational profiles of multiple single CTCs and cfDNA isolated from the same blood samples taken from patients with metastatic breast cancer (MBC). MBC patient blood samples were enriched for EpCAMpositive CTCs by CellSearch ${ }^{\mathrm{TM}}$ and a count obtained for each sample. In 5 patients with $\geq 100$ CTCs per $7.5 \mathrm{ml}$ blood, multiple single CTCs were isolated by DEPArray ${ }^{\mathrm{TM}}$. Next-generation sequencing (NGS) of $\sim 2200$ mutations in 50 known cancer genes was performed on isolated single CTCs, cfDNA from the same blood sample, and matched primary tumour tissue. NGS analysis of 40 individual CTCs from 5 MBC patients revealed mutational heterogeneity in PIK3CA, TP53, ESR1, and KRAS genes between individual CTCs in 4/5 patients. In all 4 patients, matched cfDNA profiles provided an accurate reflection of mutations observed in individual CTCs; however, more mutations were detected in cfDNA than in matched CTCs. One patient had no mutations in cfDNA or CTCs. ESR1 and KRAS gene mutations were absent from primary tumour tissue and therefore likely reflect either a minor sub-clonal mutation or were acquired with disease progression. Our results demonstrate that cfDNA reflects persisting EpCAM-positive CTCs in patients with high CTC counts and may enable monitoring of the metastatic burden for clinical decision making.

Open Access This article is distributed under the terms of the Creative Commons Attribution 4.0 International License (http:// creativecommons.org/licenses/by/4.0/), which permits unrestricted use, distribution, and reproduction in any medium, provided you give appropriate credit to the original author(s) and the source, provide a link to the Creative Commons license, and indicate if changes were made. 